\title{
An optimal control problem for mean-field forward-backward stochastic differential equation with noisy observation *
}

\author{
Guangchen Wang $^{\text {a }}$, Hua Xiao ${ }^{\text {a,b }}$, Guojing Xing ${ }^{\text {a }}$ \\ ${ }^{a}$ School of Control Science and Engineering, Shandong University, Jinan 250061, China \\ ${ }^{\mathrm{b}}$ School of Mathematics and Statistics, Shandong University, Weihai 264209, China
}

\begin{abstract}
This article is concerned with an optimal control problem derived by mean-field forward-backward stochastic differential equation with noisy observation, where the drift coefficients of the state equation and the observation equation are linear with respect to the state and its expectation. The control problem is different from the existing literature on optimal control for mean-field stochastic systems, and has more applications in mathematical finance, e.g., asset-liability management problem with recursive utility, systematic risk model. Using a backward separation method with a decomposition technique, two optimality conditions along with two coupled forward-backward optimal filters are derived. Several linear-quadratic optimal control problems for mean-field forward-backward stochastic differential equations are studied. Closed-form optimal solutions are explicitly obtained in detailed situations.
\end{abstract}

Key words: Backward separation method; closed-form optimal solution; maximum principle; mean-field forward-backward stochastic differential equation; optimal filter; recursive utility.

\section{Introduction}

\subsection{Notation}

We denote by $T>0$ a fixed time horizon, by $\mathbb{R}^{m}$ the $m$-dimensional Euclidean space, by $|\cdot|($ resp. $\langle\cdot, \cdot\rangle)$ the norm (resp. scalar product) in a Euclidean space, by $A^{\top}$ (resp. $A^{-1}$ ) the transposition (resp. reverse) of $A$, by $S^{m}$ the set of symmetric $m \times m$ matrices with real elements, by $f_{x}$ the partial derivative of $f$ with respect to $x$, and by $C$ a positive constant, which can be different from line to line. Let $\left(\Omega, \mathscr{F},\left(\mathscr{F}_{t}\right)_{0<t<T}, \mathbb{P}\right)$ be a complete filtered probability space, on which are given an $\mathscr{F}_{t}$-adapted standard Brownian motion $\left(w_{t}, \tilde{w}_{t}\right)$ with values in $\mathbb{R}^{r+\tilde{r}}$ and a Gaussian random variable $\xi$ with

\footnotetext{
* This work is supported in part by the NSF of China under Grants 11371228, 61422305, 61304130 and 61573217, by the NSF for Distinguished Young Scholars of Shandong Province of China under Grant JQ201418, and by the Research Fund for the Taishan Scholar Project of Shandong Province of China. The material in this work was partially presented at the 35th Chinese Control Conference, July 27-29, 2016, Chengdu. Corresponding author: Hua Xiao.

Email addresses: wguangchen@sdu.edu.cn (Guangchen Wang), xiao_hua@sdu.edu.cn (Hua Xiao), xgjsdu@sdu.edu.cn (Guojing Xing).
}

mean $\mu_{0}$ and covariance matrix $\sigma_{0} .(w, \tilde{w})$ is independent of $\xi$. If $A \in S^{m}$ is positive (semi) definite, we write $A>(\geq) 0$. If $x:[0, T] \rightarrow \mathbb{R}^{m}$ is uniformly bounded, we write $x \in \mathscr{L}^{\infty}\left(0, T ; \mathbb{R}^{m}\right)$. If $x: \Omega \rightarrow \mathbb{R}^{m}$ is an $\mathscr{F}_{T^{-}}$ measurable, square-integrable random variable, we write $x \in \mathscr{L}_{\mathscr{F}}^{2}\left(\mathbb{R}^{m}\right)$. If $x:[0, T] \times \Omega \rightarrow \mathbb{R}^{m}$ is an $\mathscr{F}_{t}$-adapted, square-integrable process, we write $x \in \mathscr{L}_{\mathscr{F}}^{2}\left(0, T ; \mathbb{R}^{m}\right)$. We also adopt similar notations for other processes, Euclidean spaces and filtrations.

\subsection{Motivation}

Now consider an asset-liability management problem of a firm. Let the dimension $n=k=r=\tilde{r}=1$. Denote by $\mathbb{E}$ the expectation with respect to $\mathbb{P}$, by $v_{t}$ the control strategy of the firm, by $x_{t}^{v}$ the cash-balance, and by $\bar{l}_{t}^{v}$ the liability process. Norberg [35] described the liability process by a Brownian motion with drift. The model, however, is not just the one we want. In fact, it is possible that the control strategy and the mean of the cash-balance can influence the liability process, due to the complexity of the financial market and the risk aversion behavior of the firm. Such an example can be found in Huang et al. [22], where the liability process depends on a control strategy (for example, capital injection or withdrawal) of the firm. Along this line, we proceed to 
improve the liability process here. Suppose that $\bar{l}_{t}^{v}$ satisfies a linear stochastic differential equation (SDE, in short) of the form

$$
-d \bar{l}_{t}^{v}=\left(\bar{a}_{t} \mathbb{E} x_{t}^{v}+b_{t} v_{t}+\bar{b}_{t}\right) d t+c_{t} d w_{t} .
$$

Here $\bar{a}, b, \bar{b}, c, a, f, g$ and $h$ are deterministic and uniformly bounded. $\bar{b}_{t}$ and $c_{t}$ denote the liability rate and the volatility coefficient, respectively. Suppose that the firm owns an initial investment $\xi$, and only invests in a money account with the compounded interest rate $a_{t}$. Then the cash-balance of the firm is

$$
x_{t}^{v}=e^{\int_{0}^{t} a_{s} d s}\left(\xi-\int_{0}^{t} e^{-\int_{0}^{s} a_{r} d r} d \bar{l}_{s}^{v}\right) .
$$

It follows from Itô's formula that

$$
\left\{\begin{aligned}
d x_{t}^{v} & =\left(a_{t} x_{t}^{v}+\bar{a}_{t} \mathbb{E} x_{t}^{v}+b_{t} v_{t}+\bar{b}_{t}\right) d t+c_{t} d w_{t} \\
x_{0}^{v} & =\xi
\end{aligned}\right.
$$

Note that, if $b_{t}=1, \bar{b}_{t}=0, a_{t}=-\bar{a}_{t}=$ const. and $c_{t}=$ const., then the cash-balance equation is just the systematic risk model of inter-bank borrowing and lending introduced in Carmona et al. [11]. Besides the systematic risk model, the equation can also be reduced to an air conditioning control model in energy-efficient buildings. See, e.g., Example 2 in Djehiche et al. [12] for more details.

Due to the discreteness of account information, it is possible for the firm to partially observe the cash-balance by the stock price

$$
\left\{\begin{aligned}
d S_{t}^{v} & =S_{t}^{v}\left[\left(f_{t} x_{t}^{v}+g_{t}+\frac{1}{2} h_{t}^{2}\right) d t+h_{t} d \tilde{w}_{t}\right], \\
S_{0}^{v} & =1 .
\end{aligned}\right.
$$

Set $Y_{t}^{v}=\log S_{t}^{v}$. It holds that $Y^{v}$ is governed by

$$
\left\{\begin{aligned}
d Y_{t}^{v} & =\left(f_{t} x_{t}^{v}+g_{t}\right) d t+h_{t} d \tilde{w}_{t} \\
Y_{0}^{v} & =0
\end{aligned}\right.
$$

Suppose that the firm has triple performance objectives. The first two ones are to minimize the total cost of $v$ over $[0, T]$ and to minimize the risk of $x_{T}^{v}$. Assume that the risk is measured by $\mathbb{E}\left[\left(x_{T}^{v}-\mathbb{E} x_{T}^{v}\right)^{2}\right]$. The third one is to maximize the utility $y_{t}^{v}$ resulting from $v$. Without loss of generality, define

$$
y_{t}^{v}=\mathbb{E}\left[x_{T}^{v}+\int_{t}^{T} G\left(s, y_{s}^{v}, v_{s}\right) d s \mid \mathscr{F}_{t}\right],
$$

where $G$ is Lipschitiz continuous with respect to $(y, v)$, and $G(s, 0,0) \in \mathscr{L}_{\mathscr{F}}^{2}(0, T ; \mathbb{R})$ for $0 \leq s \leq T$. We emphasize that the current utility $y_{t}^{v}$ depends not only on the instantaneous control $v_{t}$, but also on the future utility and control $\left(y_{s}^{v}, v_{s}\right), t \leq s \leq T$. This shows the difference between the utility $y^{v}$ and the standard additive utility, and hence, $y^{v}$ is called as a stochastic differential recursive utility in Duffie and Epstein [13]. Then the asset-liability management problem with recursive utility is stated as follows.

Problem (AL). Find a $\sigma\left\{Y_{s}^{v} ; 0 \leq s \leq t\right\}$-adapted and square-integrable process $v_{t}$ such that

$$
J[v]=\frac{1}{2} \mathbb{E}\left[\int_{0}^{T} B_{t} v_{t}^{2} d t+H\left(x_{T}^{v}-\mathbb{E} x_{T}^{v}\right)^{2}-2 N y_{0}^{v}\right]
$$

is minimized. Here $B>0$ and $B^{-1}$ are deterministic and uniformly bounded. $H$ and $N$ are non-negative constants. $y_{0}^{v}$ is the value of $y_{t}^{v}$ at time 0 .

Let us now turn to the recursive utility $y_{t}^{v}$ again. According to El Karoui et al. [14], $y_{t}^{v}$ admits the backward stochastic differential equation (BSDE, in short)

$$
\left\{\begin{aligned}
-d y_{t}^{v} & =G\left(t, y_{t}^{v}, v_{t}\right) d t-z_{t}^{v} d w_{t}-\tilde{z}_{t}^{v} d \tilde{w}_{t} \\
y_{T}^{v} & =x_{T}^{v}
\end{aligned}\right.
$$

With the BSDE, Problem (AL) can be rewritten as an optimal control problem derived by forward-backward stochastic differential equation (FBSDE, in short) with noisy observation. It is possible to work out one more asset-liability management problem. We omit the details to limit the length of this article.

\subsection{Problem statement}

Motivated by the examples, we study an optimal control problem for FBSDE with noisy observation. Consider a controlled FBSDE

$$
\left\{\begin{aligned}
d x_{t}^{v}= & \left(a_{t} x_{t}^{v}+\bar{a}_{t} \mathbb{E} x_{t}^{v}+b\left(t, v_{t}\right)\right) d t+c_{t} d w_{t}, \\
-d y_{t}^{v}= & \left(\alpha_{t} x_{t}^{v}+\bar{\alpha}_{t} \mathbb{E} x_{t}^{v}+\beta_{t} y_{t}^{v}+\bar{\beta}_{t} \mathbb{E} y_{t}^{v}+\gamma_{t} z_{t}^{v}\right. \\
& \left.+\bar{\gamma}_{t} \mathbb{E} z_{t}^{v}+\tilde{\gamma}_{t} \tilde{z}_{t}^{v}+\bar{\gamma}_{t} \mathbb{E} \tilde{z}_{t}^{v}+\psi\left(t, v_{t}\right)\right) d t \\
& -z_{t}^{v} d w_{t}-\tilde{z}_{t}^{v} d \tilde{w}_{t}, \\
x_{0}^{v}= & \xi, \quad y_{T}^{v}=\rho x_{T}^{v}+\bar{\rho} \mathbb{E} x_{T}^{v},
\end{aligned}\right.
$$

where $\left(x^{v}, y^{v}, z^{v}, \tilde{z}^{v}\right)$ is the sate, $v$ is the control, and $(w, \tilde{w})$ is the Brownian motion. Since the mean of the state influences the state equation, we call the equation a mean-field FBSDE, or a McKean-Vlasov FBSDE. Assume that $\left(x^{v}, y^{v}, z^{v}, \tilde{z}^{v}\right)$ is partially observed through

$$
\left\{\begin{aligned}
d Y_{t}^{v} & =\left(f_{t} x_{t}^{v}+\bar{f}_{t} \mathbb{E} x_{t}^{v}+g\left(t, v_{t}\right)\right) d t+h_{t} d \tilde{w}_{t}, \\
Y_{0}^{v} & =0 .
\end{aligned}\right.
$$


The cost functional is

$J[v]=\mathbb{E}\left[\int_{0}^{T} l\left(t, x_{t}^{v}, \mathbb{E} x_{t}^{v}, v_{t}\right) d t+\phi\left(x_{T}^{v}, \mathbb{E} x_{T}^{v}\right)+\varphi\left(y_{0}^{v}\right)\right]$.

Here $v_{t}$ is required to be $\sigma\left\{Y_{s}^{v} ; 0 \leq s \leq t\right\}$-adapted and to satisfy $\mathbb{E} \sup _{0 \leq t \leq T}\left|v_{t}\right|^{2}<+\infty . a, \bar{a}, b, c, \alpha, \bar{\alpha}, \beta, \bar{\beta}$, $\gamma, \bar{\gamma}, \tilde{\gamma}, \overline{\tilde{\gamma}}, \psi, \rho, \bar{\rho}, f, \bar{f}, g, h, l, \phi$ and $\varphi$ will be specified in Section 2. Our problem is to select an admissible control $v$ to minimize $J[v]$. We denote the problem by Problem (MFC), where "MF" and "C" are the capital initials of "mean-field" and "control", respectively.

To solve Problem (MFC), it is natural to use dynamic programming and maximum principle. The dynamic programming, however, does not hold even if the BSDE and the observation equation are not present, mainly due to the inclusion of the mean of the state, which leads to the time inconsistency. We instead study the maximum principle for optimality of Problem (MFC).

\subsection{Briefly historical retrospect and contribution of this paper}

Mean-field theory provides an effective tool for investigating the collective behaviors arising from individuals' mutual interactions in various different fields, say, finance, game, engineering. Since the independent introduction by Lasry and Lions [27] and Huang et al. [24,25], the mean-field theory has attracted more and more attention. Let us now briefly recall some latest developments which are related to Problem (MFC).

Although the study of mean-field SDE has a long history with the pioneering works of Kac [26] and McKean [31], mean-field type control is a rather new research direction. In 2001, Ahmed and Ding [1] used the Nisio nonlinear operator semigroup to obtain an extended dynamic programming. By dual techniques, maximum principles for several mean-field SDEs with full information were derived. See, e.g., Buckdahn et al. [7], Li [28], Hafayed and Abbas [16], Shen et al. [37], Djehiche et al. [12]. Subsequently, Meyer-Brandis et al. [32], Hafayed et al. $[17,18]$ studied the partial information case, where noisy observation and filtering are excluded. As applications of the derived maximum principles, $[32,41,20]$ solved mean-variance problems with full and partial information. Yong [43] studied a linear-quadratic (LQ, in short) optimal control problem for mean-field SDE with full information. Further, Yong [44] investigated the timeinconsistency feature of the LQ problem, and obtained both open-loop and closed-loop equilibrium solutions. Later, Huang et al. [21] extended the LQ problem to the case of infinite horizon. For the discrete-time counterpart of the LQ problem, please refer to Elliott et al. [15], $\mathrm{Ni}$ et al. $[33,34]$ and the references therein for more details. It is worth pointing out that the investigation of mean-field type control is also partially inspired by the interest in mean-field game. If we only focus on a single decision maker, also called a representative agent, mean-field game can be regarded as mean-field type control. Generally speaking, an exact Nash equilibrium for mean-filed game with a large number of decision makers is rarely available except for special cases (see, e.g., Carmona et al. [11]). It is highly desirable to find a good approximation of this Nash equilibrium. Please refer to Carmona et al. [10], Tembine et al. [38], Bensoussan et al. [4], etc. for more details on different types of approximation equilibrium. See also Bensoussan et al. [6] for a comprehensive study of a general LQ mean field game.

Both mean-field type control and mean-field game lead to mean-field FBSDE. Buckdahn et al. [8] studied the well-posedness of a decoupled mean-field FBSDE using a limit approach. Bensoussan et al. [5], Carmona and Delarue [9] extended [8] to the fully coupled mean-field FBSDE case in terms of a continuation method introduced in Peng and $\mathrm{Wu}$ [36]. Mean-field FBSDE is a welldefined dynamic system, it is very natural and appealing to study control and game problems for mean-field FBSDEs as well as their applications. To our knowledge, there is only a few literature on this topic. For example, $\mathrm{Li}$ and Liu [29] studied an optimal control problem for fully coupled mean-field FBSDE. Hafayed et al. [19] obtained a maximum principle for mean-field FBSDE with jump. Huang et al. [23] studied an LQ game with a linear mean-field BSDE system and a quadratic cost functional. $[19,23]$ also provided some applications in meanvariance and recursive utility problems.

In this paper, we are interested in studying Problem (MFC). Compared with the above literature, this problem has several new features as follows.

- The state $\left(x^{v}, y^{v}, z^{v}, \tilde{z}^{v}\right)$ satisfies a mean-field FBSDE rather than a mean-field SDE, and is only partially observed by a noisy process. This endows Problem (MFC) more practical meanings in reality.

- Unlike those control models solved in Bensoussan [3], the classical separation principle does not work here, mainly due to the fact that the mean square error of filtering of BSDE depends on the control in general.

- The state equation involves the mean of the state, and thus, Problem (MFC) can not be studied by transforming it into a standard control problem for FBSDE. This feature can be supported by Example 2.2 in this paper.

There is a few papers related to Problem (MFC). Let us make a brief comment on them. Wang et al. [42] posted a partially observable mean-field type optimal control problem for SDE. They used a backward separation method and a probability transformation to decouple a circular dependence between the control and the observation first, and then derived a necessary condition for optimality. The result was further generalized 
in Wang et al. [41] by the backward separation method with an approximation technique. Later, $\mathrm{Hu}$ et al. [20] studied an optimal control problem for mean-field SDE with jump. Zhang [45] addressed the case with correlated state and observation noises. We emphasize that the approach applied in $[20,41,42,45]$ is based on at least one of the assumptions below.

- The state satisfies an SDE rather than an FBSDE.

- The drift term of the observation equation is uniformly bounded with respect to the state and the control.

- The control has no effect on the observation.

- The control $v$ satisfies $\mathbb{E} \sup _{0 \leq t \leq T}\left|v_{t}\right|^{\ell}<+\infty, \forall \ell>0$.

Clearly, Problem (MFC) does not meet these assumptions. Another approach is desired to develop to address Problem (MFC). In [40], Wang et al. studied an LQ control problem for classical FBSDE (i.e., the dynamics of the FBSDE does not depend on the probability distribution of the state). Inspired by Bensoussan [2], they solved the LQ problem by combining a decomposition technique with the backward separation method. Recently, our further study on the approach provided in Wang et al. [40] finds out the availability of the approach to some nonlinear control problems with noisy observations, say, Problem (MFC). In this paper, we will show how to use the approach to address Problem (MFC). See also Wang et al. [39] for other developments about partially observable optimal control for FBSDE.

The rest of this article is organized as follows. In Section 2 , we carefully formulate Problem (MFC) first, and then provide illustrative examples and preliminary results. In Section 3, we obtain two optimality conditions and two coupled forward-backward optimal filtering equations. In Section 4, we study an LQ case of Problem (MFC) and obtain a feedback representation of optimal control. In Section 5, we explicitly solve an asset-liability management problem with noisy observation, and work out an illustrative numerical example. Some concluding remarks and proofs of the preliminary results are given in Section 6 and Appendix, respectively.

\section{Problem formulation and preliminary}

One difficulty to study Problem (MFC) is there is a circular dependence between the control $v$ and the observation $Y^{v}$, which results in the unavailability of classical variation. Here we will adopt a decomposition technique, similar to those of $[2,40]$, to overcome the difficulty. De- fine $\left(x^{0}, y^{0}, z^{0}, \tilde{z}^{0}\right)$ and $Y^{0}$ by

$$
\left\{\begin{aligned}
d x_{t}^{0}= & \left(a_{t} x_{t}^{0}+\bar{a}_{t} \mathbb{E} x_{t}^{0}\right) d t+c_{t} d w_{t}, \\
-d y_{t}^{0}= & \left(\alpha_{t} x_{t}^{0}+\bar{\alpha}_{t} \mathbb{E} x_{t}^{0}+\beta_{t} y_{t}^{0}+\bar{\beta}_{t} \mathbb{E} y_{t}^{0}+\gamma_{t} z_{t}^{0}\right. \\
& \left.+\bar{\gamma}_{t} \mathbb{E} z_{t}^{0}+\tilde{\gamma}_{t} \tilde{z}_{t}^{0}+\overline{\tilde{\gamma}}_{t} \mathbb{E} \tilde{z}_{t}^{0}\right) d t \\
& -z_{t}^{0} d w_{t}-\tilde{z}_{t}^{0} d \tilde{w}_{t}, \\
x_{0}^{0}= & \xi, \quad y_{T}^{v}=\rho x_{T}^{0}+\bar{\rho} \mathbb{E} x_{T}^{0},
\end{aligned}\right.
$$

and

$$
\left\{\begin{aligned}
d Y_{t}^{0} & =\left(f_{t} x_{t}^{0}+\bar{f}_{t} \mathbb{E} x_{t}^{0}\right) d t+h_{t} d \tilde{w}_{t}, \\
Y_{0}^{0} & =0,
\end{aligned}\right.
$$

where $a, \bar{a} \in \mathscr{L}^{\infty}\left(0, T ; \mathbb{R}^{n \times n}\right), c \in \mathscr{L}^{\infty}\left(0, T ; \mathbb{R}^{n \times r}\right)$, $\alpha, \bar{\alpha} \in \mathscr{L}^{\infty}\left(0, T ; \mathbb{R}^{m \times n}\right), \beta, \bar{\beta} \in \mathscr{L}^{\infty}\left(0, T ; \mathbb{R}^{m \times m}\right), f$, $\bar{f} \in \mathscr{L}^{\infty}\left(0, T ; \mathbb{R}^{\tilde{r} \times n}\right), h, h^{-1} \in \mathscr{L}^{\infty}\left(0, T ; \mathbb{R}^{\tilde{r} \times \tilde{r}}\right), \underline{\underline{\gamma}}=$ $\left(\underline{\gamma}_{1}, \cdots, \underline{\gamma}_{r}\right), \bar{\gamma}=\left(\bar{\gamma}_{1}, \cdots, \bar{\gamma}_{r}\right), \tilde{\gamma}=\left(\tilde{\gamma}_{1}, \cdots, \tilde{\gamma}_{\tilde{r}}\right), \overline{\tilde{\gamma}}=$ $\left(\tilde{\tilde{\gamma}}_{1}, \cdots, \overline{\tilde{\gamma}}_{\tilde{r}}\right), z^{0}=\left(z_{1}^{0}, \cdots, z_{r}^{0}\right), \tilde{z}^{0}=\left(\tilde{z}_{1}^{0}, \cdots, \tilde{z}_{\tilde{r}}^{0}\right), \gamma_{j}$ $\bar{\gamma}_{j}, \tilde{\gamma}_{j}, \overline{\tilde{\gamma}}_{j} \in \mathscr{L}^{\infty}\left(0, T ; \mathbb{R}^{m \times m}\right)$ for $j=1, \cdots, r$ or $\tilde{r}$, and $\rho, \bar{\rho} \in \mathbb{R}^{m \times n}$ are constant matrices. Here we use the simplified notation $\gamma_{t} z_{t}^{0} \triangleq \sum_{j=1}^{r} \gamma_{j t} z_{j t}^{0}$. Similarly, it is also applicable for the notations $\tilde{\gamma}_{t} \tilde{z}_{t}^{0}, \bar{\gamma}_{t} \mathbb{E} z_{t}^{0}, \overline{\tilde{\gamma}}_{t} \mathbb{E} \tilde{z}_{t}^{0}, \cdots$.

Let $v \in \mathscr{L}_{\mathscr{F}}^{2}\left(0, T ; \mathbb{R}^{k}\right)$ be a control process. Define $\left(x^{v, 1}, y^{v, 1}, z^{v, 1}, \tilde{z}^{v, 1}\right)$ and $Y^{v, 1}$ by

$$
\left\{\begin{aligned}
\dot{x}_{t}^{v, 1}= & a_{t} x_{t}^{v, 1}+\bar{a}_{t} \mathbb{E} x_{t}^{v, 1}+b\left(t, v_{t}\right), \\
-d y_{t}^{v, 1}= & \left(\alpha_{t} x_{t}^{v, 1}+\bar{\alpha}_{t} \mathbb{E} x_{t}^{v, 1}+\beta_{t} y_{t}^{v, 1}+\bar{\beta}_{t} \mathbb{E} y_{t}^{v, 1}\right. \\
& +\gamma_{t} z_{t}^{v, 1}+\bar{\gamma}_{t} \mathbb{E} z_{t}^{v, 1}+\tilde{\gamma}_{t} \tilde{z}_{t}^{v, 1}+\overline{\tilde{\gamma}}_{t} \mathbb{E} \tilde{z}_{t}^{v, 1} \\
& \left.+\psi\left(t, v_{t}\right)\right) d t-z_{t}^{v, 1} d w_{t}-\tilde{z}_{t}^{v, 1} d \tilde{w}_{t}, \\
x_{0}^{v, 1}= & \xi, \quad y_{T}^{v, 1}=\rho x_{T}^{v, 1}+\bar{\rho} \mathbb{E} x_{T}^{v, 1}
\end{aligned}\right.
$$

and

$$
\left\{\begin{array}{l}
\dot{Y}_{t}^{v, 1}=f_{t} x_{t}^{v, 1}+\bar{f}_{t} \mathbb{E} x_{t}^{v, 1}+g\left(t, v_{t}\right), \\
Y_{0}^{v, 1}=0
\end{array}\right.
$$

where $g:[0, T] \times \mathbb{R}^{k} \rightarrow \mathbb{R}^{\tilde{r}}$ satisfies $\mathbb{E} \int_{0}^{T}\left|g\left(t, v_{t}\right)\right|^{2} d t<$ $+\infty, b:[0, T] \times \mathbb{R}^{k} \rightarrow \mathbb{R}^{n}$ and $\psi:[0, T] \times \mathbb{R}^{k} \rightarrow \mathbb{R}^{m}$ are continuous and continuously differentiable with respect to $t$ and $v$, respectively, $b_{v} \in \mathscr{L}^{\infty}\left(0, T ; \mathbb{R}^{n \times k}\right)$ and $\psi_{v} \in$ $\mathscr{L}^{\infty}\left(0, T ; \mathbb{R}^{m \times k}\right)$. Since (1) and (3) are decoupled, it is easy to see from Buckdahn et al. [8] that (1), (2), (3) and (4) have unique solutions, respectively. Define

$$
\begin{array}{ll}
x_{t}^{v}=x_{t}^{0}+x_{t}^{v, 1}, & y_{t}^{v}=y_{t}^{0}+y_{t}^{v, 1}, \\
z_{t}^{v}=z_{t}^{0}+z_{t}^{v, 1}, & \tilde{z}_{t}^{v}=\tilde{z}_{t}^{0}+\tilde{z}_{t}^{v, 1}
\end{array}
$$

and

$$
Y_{t}^{v}=Y_{t}^{0}+Y_{t}^{v, 1} .
$$

It follows from Itô's formula that $\left(x^{v}, y^{v}, z^{v}, z^{v}\right)$ and $Y^{v}$ 
uniquely solve

$$
\left\{\begin{aligned}
d x_{t}^{v}= & \left(a_{t} x_{t}^{v}+\bar{a}_{t} \mathbb{E} x_{t}^{v}+b\left(t, v_{t}\right)\right) d t+c_{t} d w_{t}, \\
-d y_{t}^{v}= & \left(\alpha_{t} x_{t}^{v}+\bar{\alpha}_{t} \mathbb{E} x_{t}^{v}+\beta_{t} y_{t}^{v}+\bar{\beta}_{t} \mathbb{E} y_{t}^{v}+\gamma_{t} z_{t}^{v}\right. \\
& \left.+\bar{\gamma}_{t} \mathbb{E} z_{t}^{v}+\tilde{\gamma}_{t} \tilde{z}_{t}^{v}+\tilde{\tilde{\gamma}}_{t} \mathbb{E} \tilde{z}_{t}^{v}+\psi\left(t, v_{t}\right)\right) d t \\
& -z_{t}^{v} d w_{t}-\tilde{z}_{t}^{v} d \tilde{w}_{t} \\
x_{0}^{v}= & \xi, \quad y_{T}^{v}=\rho x_{T}^{v}+\bar{\rho} \mathbb{E} x_{T}^{v},
\end{aligned}\right.
$$

and

$$
\left\{\begin{aligned}
d Y_{t}^{v} & =\left(f_{t} x_{t}^{v}+\bar{f}_{t} \mathbb{E} x_{t}^{v}+g\left(t, v_{t}\right)\right) d t+h_{t} d \tilde{w}_{t} \\
Y_{0}^{v} & =0
\end{aligned}\right.
$$

respectively. Let $\mathscr{F}_{t}^{Y^{v}}=\sigma\left\{Y_{s}^{v} ; 0 \leq s \leq t\right\}$ and $\mathscr{F}_{t}^{Y^{0}}=$ $\sigma\left\{Y_{s}^{0} ; 0 \leq s \leq t\right\}$. Note that the first observable filtration depends on the control $v$. However, the second one is not the case. We now give a definition of admissible control. Let $U$ be a nonempty convex subset of $\mathbb{R}^{k}$, and let $\mathscr{U}_{\text {ad }}^{0}$ be the collection of all $\mathscr{F}_{t}^{Y^{0}}$-adapted processes with values in $U$ such that $\mathbb{E} \sup _{0 \leq t \leq T}\left|v_{t}\right|^{2}<+\infty$.

Definition 2.1. A control $v$ is called admissible, if $v \in$ $\mathscr{U}_{a d}^{0}$ is $\mathscr{F}_{t}^{Y^{v}}$-adapted. The set of all admissible controls is denoted by $\mathscr{U}_{a d}$.

With the definition, it follows from the equality (6) that

Proposition 2.1. For any $v \in \mathscr{U}_{a d}, \mathscr{F}_{t}^{Y^{v}}=\mathscr{F}_{t}^{Y^{0}}$.

It implies that the control $v$ has no effect on the observation $Y^{v}$, i.e., the circular dependence between $v$ and $Y^{v}$ is decoupled.

The cost functional is in the form of

$$
\begin{aligned}
J[v]= & \mathbb{E}\left[\int_{0}^{T} l\left(t, x_{t}^{v}, \mathbb{E} x_{t}^{v}, v_{t}\right) d t\right. \\
& \left.+\phi\left(x_{T}^{v}, \mathbb{E} x_{T}^{v}\right)+\varphi\left(y_{0}^{v}\right)\right],
\end{aligned}
$$

where $l:[0, T] \times \mathbb{R}^{n+n} \times U \rightarrow \mathbb{R}, \phi: \mathbb{R}^{n+n} \rightarrow \mathbb{R}$ and $\varphi: \mathbb{R}^{m} \rightarrow \mathbb{R}$ are continuously differentiable with respect to $(x, \bar{x}, v),(x, \bar{x})$ and $y$, respectively, and there is a constant $C>0$ such that

$$
\begin{aligned}
|\phi(x, \bar{x})| & \leq C\left(1+|x|^{2}+|\bar{x}|^{2}\right), \\
\left|\phi_{x}(x, \bar{x})\right|+\left|\phi_{\bar{x}}(x, \bar{x})\right| & \leq C(1+|x|+|\bar{x}|), \\
|l(t, x, \bar{x}, v)| & \leq C\left(1+|x|^{2}+|\bar{x}|^{2}+|v|^{2}\right), \\
\left|l_{\chi}(t, x, \bar{x}, v)\right| & \leq C(1+|x|+|\bar{x}|+|v|), \\
|\varphi(y)| & \leq C\left(1+|y|^{2}\right), \\
\left|\varphi_{y}(y)\right| & \leq C\left(1+|y|^{2}\right)
\end{aligned}
$$

with $\chi=x, \bar{x}, v$. Then the optimal control problem for mean-field FBSDE is restated as follows.
Problem (MFC). Find a $u \in \mathscr{U}_{a d}$ such that $J[u]=$ $\inf _{v \in \mathscr{U}_{a d}} J[v]$ subject to (7), (8) and (9). Any $u$ satisfying the equality is called an optimal control of Problem (MFC), and $\left(x^{u}, y^{u}, z^{u}, \tilde{z}^{u}\right)$ and $J[u]$ are called the optimal state and the optimal cost functional, respectively.

Note that the above decomposition technique is restricted to special structures of state and observation equations, say, the case that (7) and (8) are linear with respect to $\left(x^{v}, y^{v}, z^{v}, \tilde{z}^{v}\right)$, the diffusion coefficient of $(7)$ is deterministic, and the drift coefficient of (8) is independent of $\left(y^{v}, z^{v}, \tilde{z}^{v}\right)$. It is worth investigating the availability of the technique to decompose more general state and observation equations in the future.

Next, let us show more new features of Problem (MFC) by two simple examples. Roughly speaking, Example 2.1 tells us that Problem (MFC) is possibly applied to solve a partially observable optimal control problem for mean-field SDE with stochastic coefficients in certain situations; Example 2.2 reveals that Problem (MFC) is not a trivial extension to a partially observable optimal control problem for FBSDE without mean-field term.

Example 2.1. Let $\alpha_{t}=\bar{\alpha}_{t}=\beta_{t}=\bar{\beta}_{t}=\bar{\gamma}_{t}=\overline{\tilde{\gamma}}_{t}=0$ in Problem (MFC). Then (7) is reduced to

$$
\left\{\begin{aligned}
d x_{t}^{v} & =\left(a_{t} x_{t}^{v}+\bar{a}_{t} \mathbb{E} x_{t}^{v}+b\left(t, v_{t}\right)\right) d t+c_{t} d w_{t}, \\
-d y_{t}^{v} & =\left(\gamma_{t} z_{t}^{v}+\tilde{\gamma}_{t} \tilde{z}_{t}^{v}+\psi\left(t, v_{t}\right)\right) d t-z_{t}^{v} d w_{t}-\tilde{z}_{t}^{v} d \tilde{w}_{t}, \\
x_{0}^{v} & =\xi, \quad y_{T}^{v}=\rho x_{T}^{v}+\bar{\rho} \mathbb{E} x_{T}^{v} .
\end{aligned}\right.
$$

Solving the BSDE in (10), we get

$$
y_{0}^{v}=\mathbb{E}\left[\left\langle\eta_{T}, \rho x_{T}^{v}+\bar{\rho} \mathbb{E} x_{T}^{v}\right\rangle+\int_{0}^{T}\left\langle\eta_{t}, \psi\left(t, v_{t}\right)\right\rangle d t\right]
$$

with

$$
\left\{\begin{aligned}
d \eta_{t} & =\gamma_{t} \eta_{t} d w_{t}+\tilde{\gamma}_{t} \eta_{t} d \tilde{w}_{t} \\
\eta_{0} & =I_{m}
\end{aligned}\right.
$$

where $I_{m}$ is an $m$-dimensional vector with all components being 1 . Plugging (11) into (9), we have

$$
\begin{aligned}
\mathcal{J}[v]= & \mathbb{E}\left[\int_{0}^{T} l\left(t, x_{t}^{v}, \mathbb{E} x_{t}^{v}, v_{t}\right) d t+\phi\left(x_{T}^{v}, \mathbb{E} x_{T}^{v}\right)\right. \\
& +\varphi\left(\mathbb { E } \left[\left\langle\eta_{T}, \rho x_{T}^{v}+\bar{\rho} \mathbb{E} x_{T}^{v}\right\rangle\right.\right. \\
& \left.\left.\left.+\int_{0}^{T}\left\langle\eta_{t}, \psi\left(t, v_{t}\right)\right\rangle d t\right]\right)\right] .
\end{aligned}
$$

Then Problem (MFC) is reduced to minimize $\mathcal{J}[v]$ over $\mathscr{U}_{a d}$ subject to (8) and the SDE in (10). It is worth noting that we start with a control model with deterministic coefficients, but we end up with a control model with stochastic coefficients. The interesting phenomena is caused by the introduction of the BSDE in (10). Just because of this, maybe it provides a potential method to 
investigate a control problem for mean-field SDE with stochastic coefficients under certain conditions, i.e., we can change it into an equivalent control problem for mean-field FBSDE with deterministic coefficients. The details of how to make use of this potential method will be shown in our future publications, because they beyond the scope of the present paper.

Example 2.2. Find a $v \in \mathscr{U}_{a d}$ such that

$$
\begin{aligned}
J[v]=\mathbb{E} & {\left[\int _ { 0 } ^ { T } \left(\left\langle A_{t} x_{t}^{v}, x_{t}^{v}\right\rangle+\left\langle\bar{A}_{t} \mathbb{E} x_{t}^{v}, \mathbb{E} x_{t}^{v}\right\rangle\right.\right.} \\
& \left.+\left\langle B_{t} v_{t}, v_{t}\right\rangle\right) d t+\left\langle H x_{T}^{v}, x_{T}^{v}\right\rangle \\
& \left.+\left\langle\bar{H} \mathbb{E} x_{T}^{v}, \mathbb{E} x_{T}^{v}\right\rangle+\left\langle M y_{0}^{v}, y_{0}^{v}\right\rangle\right]
\end{aligned}
$$

is minimized, subject to (7) and (8) with the assumption $b\left(t, v_{t}\right)=\tilde{b}_{t} v_{t}+\bar{b}_{t}, \psi\left(t, v_{t}\right)=\tilde{\psi}_{t} v_{t}+\bar{\psi}_{t}, g\left(t, v_{t}\right)=\tilde{g}_{t} v_{t}+$ $\bar{g}_{t}$, where $A, \bar{A} \in \mathscr{L}^{\infty}\left(0, T ; S^{n}\right), B \in \mathscr{L}^{\infty}\left(0, T ; S^{k}\right)$, $H, \bar{H} \in S^{n}, M \in S^{m}, A, B, H, M \geq 0, A+\bar{A}, H+$ $\bar{H} \geq 0, \tilde{b} \in \mathscr{L}^{\infty}\left(0, T ; \mathbb{R}^{n \times k}\right), \bar{b} \in \mathscr{L}^{\infty}\left(0, T ; \mathbb{R}^{n}\right)$, $\tilde{\psi} \in \mathscr{L}^{\infty}\left(0, T ; \mathbb{R}^{m \times k}\right), \bar{\psi} \in \mathscr{L}^{\infty}\left(0, T ; \mathbb{R}^{m}\right), \tilde{g} \in$ $\mathscr{L}^{\infty}\left(0, T ; \mathbb{R}^{\tilde{r} \times k}\right)$ and $\bar{g} \in \mathscr{L}^{\infty}\left(0, T ; \mathbb{R}^{\tilde{r}}\right)$. For simplicity, we denote the LQ problem by Example (MFLQ).

Taking expectations on both sides of (7) and (8), we have

$$
\left\{\begin{aligned}
\mathbb{E} \dot{x}_{t}^{v}= & \frac{d}{d t} \mathbb{E} x_{t}^{v}=\left(a_{t}+\bar{a}_{t}\right) \mathbb{E} x_{t}^{v}+\tilde{b}_{t} \mathbb{E} v_{t}+\bar{b}_{t} \\
-\mathbb{E} \dot{y}_{t}^{v}= & -\frac{d}{d t} \mathbb{E} y_{t}^{v}=\left(\alpha_{t}+\bar{\alpha}_{t}\right) \mathbb{E} x_{t}^{v}+\left(\beta_{t}+\bar{\beta}_{t}\right) \mathbb{E} y_{t}^{v} \\
& +\left(\gamma_{t}+\bar{\gamma}_{t}\right) \mathbb{E} z_{t}^{v}+\left(\tilde{\gamma}_{t}+\overline{\tilde{\gamma}}_{t}\right) \mathbb{E} \tilde{z}_{t}^{v} \\
& +\tilde{\psi}_{t} \mathbb{E} v_{t}+\bar{\psi}_{t} \\
\mathbb{E} x_{0}^{v}= & \mu_{0}, \quad \mathbb{E} y_{T}^{v}=(\rho+\bar{\rho}) \mathbb{E} x_{T}^{v}
\end{aligned}\right.
$$

and

$$
\left\{\begin{array}{l}
\mathbb{E} \dot{Y}_{t}^{v}=\frac{d}{d t} \mathbb{E} Y_{t}^{v}=\left(f_{t}+\bar{f}_{t}\right) \mathbb{E} x_{t}^{v}+\tilde{g}_{t} \mathbb{E} v_{t}+\bar{g}_{t}, \\
\mathbb{E} Y_{0}^{v}=0
\end{array}\right.
$$

respectively. Then

$$
\left\{\begin{aligned}
d\left(x_{t}^{v}-\mathbb{E} x_{t}^{v}\right)= & {\left[a_{t}\left(x_{t}^{v}-\mathbb{E} x_{t}^{v}\right)+\tilde{b}_{t}\left(v_{t}-\mathbb{E} v_{t}\right)\right] d t } \\
& +c_{t} d w_{t}, \\
-d\left(y_{t}^{v}-\mathbb{E} y_{t}^{v}\right)= & {\left[\alpha_{t}\left(x_{t}^{v}-\mathbb{E} x_{t}^{v}\right)+\beta_{t}\left(y_{t}^{v}-\mathbb{E} y_{t}^{v}\right)\right.} \\
& +\gamma_{t}\left(z_{t}^{v}-\mathbb{E} z_{t}^{v}\right)+\tilde{\gamma}_{t}\left(\tilde{z}_{t}^{v}-\mathbb{E} \tilde{z}_{t}^{v}\right) \\
& \left.+\tilde{\psi}_{t}\left(v_{t}-\mathbb{E} v_{t}\right)\right] d t \\
& -z_{t}^{v} d w_{t}-\tilde{z}_{t}^{v} d \tilde{w}_{t} \\
x_{0}^{v}-\mathbb{E} x_{0}^{v}= & \xi-\mu_{0} \\
y_{T}^{v}-\mathbb{E} y_{T}^{v}= & \rho\left(x_{T}^{v}-\mathbb{E} x_{T}^{v}\right)
\end{aligned}\right.
$$

$$
\left\{\begin{aligned}
d\left(Y_{t}^{v}-\mathbb{E} Y_{t}^{v}\right)= & {\left[f_{t}\left(x_{t}^{v}-\mathbb{E} x_{t}^{v}\right)+\tilde{g}_{t}\left(v_{t}-\mathbb{E} v_{t}\right)\right] d t } \\
& +h_{t} d \tilde{w}_{t}, \\
Y_{0}^{v}-\mathbb{E} Y_{0}^{v}= & 0
\end{aligned}\right.
$$

respectively. Let

$$
\begin{gathered}
\mathbf{x}_{0}=\left(\begin{array}{c}
\xi-\mu_{0} \\
\mu_{0}
\end{array}\right), \quad \mathbf{v}_{t}=\left(\begin{array}{c}
v_{t}-\mathbb{E} v_{t} \\
\mathbb{E} v_{t}
\end{array}\right), \\
\mathbf{x}_{t}^{v}=\left(\begin{array}{c}
x_{t}^{v}-\mathbb{E} x_{t}^{v} \\
\mathbb{E} x_{t}^{v}
\end{array}\right), \quad \mathbf{y}_{t}^{v}=\left(\begin{array}{c}
y_{t}^{v}-\mathbb{E} y_{t}^{v} \\
\mathbb{E} y_{t}^{v}
\end{array}\right), \\
\mathbf{z}_{t}^{v}=\left(\begin{array}{c}
z_{t}^{v}-\mathbb{E} z_{t}^{v} \\
\mathbb{E} z_{t}^{v}
\end{array}\right), \quad \tilde{\mathbf{z}}_{t}^{v}=\left(\begin{array}{c}
\tilde{z}_{t}^{v}-\mathbb{E} \tilde{z}_{t}^{v} \\
\mathbb{E} \tilde{z}_{t}^{v}
\end{array}\right), \\
\mathbf{Y}_{t}^{v}=\left(\begin{array}{c}
Y_{t}^{v}-\mathbb{E} Y_{t}^{v} \\
\mathbb{E} Y_{t}^{v}
\end{array}\right)
\end{gathered}
$$

and

$$
\begin{aligned}
& \mathbf{a}_{t}=\left(\begin{array}{cc}
a_{t} & 0 \\
0 & a_{t}+\bar{a}_{t}
\end{array}\right), \quad \mathbf{b}_{t}=\left(\begin{array}{cc}
\tilde{b}_{t} & 0 \\
0 & \tilde{b}_{t}
\end{array}\right), \quad \overline{\mathbf{b}}_{t}=\left(\begin{array}{c}
0 \\
\bar{b}_{t}
\end{array}\right), \\
& \mathbf{c}_{t}=\left(\begin{array}{c}
c_{t} \\
0
\end{array}\right), \quad \check{\alpha}_{t}=\left(\begin{array}{cc}
\alpha_{t} & 0 \\
0 & \alpha_{t}+\bar{\alpha}_{t}
\end{array}\right), \\
& \check{\beta}_{t}=\left(\begin{array}{cc}
\beta_{t} & 0 \\
0 & \beta_{t}+\bar{\beta}_{t}
\end{array}\right), \quad \check{\gamma}_{t}=\left(\begin{array}{cc}
\gamma_{t} & 0 \\
0 & \gamma_{t}+\bar{\gamma}_{t}
\end{array}\right), \\
& \check{\tilde{\gamma}}_{t}=\left(\begin{array}{cc}
\tilde{\gamma}_{t} & 0 \\
0 & \tilde{\gamma}_{t}+\overline{\tilde{\gamma}}_{t}
\end{array}\right), \quad \check{\psi}_{t}=\left(\begin{array}{cc}
\tilde{\psi}_{t} & 0 \\
0 & \tilde{\psi}_{t}
\end{array}\right), \quad \overline{\bar{\psi}}_{t}=\left(\begin{array}{c}
0 \\
\bar{\psi}_{t}
\end{array}\right) \text {, } \\
& \check{c}=\left(\begin{array}{ll}
1 & 1 \\
0 & 0
\end{array}\right), \quad \check{\rho}=\left(\begin{array}{cc}
\rho & 0 \\
0 & \rho+\bar{\rho}
\end{array}\right), \quad \mathbf{f}_{t}=\left(\begin{array}{cc}
f_{t} & 0 \\
0 & f_{t}+\bar{f}_{t}
\end{array}\right), \\
& \mathbf{g}_{t}=\left(\begin{array}{c}
0 \\
\tilde{g}_{t}
\end{array}\right), \quad \mathbf{h}_{t}=\left(\begin{array}{c}
h_{t} \\
0
\end{array}\right) \text {. }
\end{aligned}
$$

Then

$$
\begin{aligned}
& \left\{\begin{aligned}
d \mathbf{x}_{t}^{v}= & \left(\mathbf{a}_{t} \mathbf{x}_{t}^{v}+\mathbf{b}_{t} \mathbf{v}_{t}+\overline{\mathbf{b}}_{t}\right) d t+\mathbf{c}_{t} d w_{t}, \\
-d \mathbf{y}_{t}^{v}= & \left(\check{\alpha}_{t} \mathbf{x}_{t}^{v}+\check{\beta}_{t} \mathbf{y}_{t}^{v}+\check{\gamma}_{t} \mathbf{z}_{t}^{v}+\check{\tilde{\gamma}}_{t} \tilde{\mathbf{z}}_{t}^{v}+\check{\psi}_{t} \mathbf{v}_{t}\right. \\
& \left.+\check{\check{\psi}}_{t}\right) d t-\check{c} \mathbf{z}_{t}^{v} d w_{t}-\check{c}_{t}^{v} d \tilde{w}_{t}, \\
\mathbf{x}_{0}^{v}= & \mathbf{x}_{0}, \quad \mathbf{y}_{T}=\check{\rho} \mathbf{x}_{T}^{v},
\end{aligned}\right. \\
& \left\{\begin{aligned}
d \mathbf{Y}_{t}^{v} & =\left(\mathbf{f}_{t} \mathbf{x}_{t}^{v}+\mathbf{g}_{t}\right) d t+\mathbf{h}_{t} d \tilde{w}_{t} \\
\mathbf{Y}_{0}^{v} & =0
\end{aligned}\right.
\end{aligned}
$$


On the other hand, let

$$
\begin{gathered}
\mathbf{A}_{t}=\left(\begin{array}{cc}
A_{t} & 0 \\
0 & A_{t}+\bar{A}_{t}
\end{array}\right), \quad \mathbf{B}_{t}=\left(\begin{array}{cc}
B_{t} & 0 \\
0 & B_{t}
\end{array}\right), \\
\mathbf{H}=\left(\begin{array}{cc}
H & 0 \\
0 & H+\bar{H}
\end{array}\right), \quad \mathbf{M}=\left(\begin{array}{cc}
M & 0 \\
0 & M
\end{array}\right) .
\end{gathered}
$$

By simple calculations, we get

$$
\begin{aligned}
\mathbb{E}\left\{\left\langle A_{t} x_{t}^{v}, x_{t}^{v}\right\rangle+\left\langle\bar{A}_{t} \mathbb{E} x_{t}^{v}, \mathbb{E} x_{t}^{v}\right\rangle\right\} & =\mathbb{E}\left\langle\mathbf{A}_{t} \mathbf{x}_{t}^{v}, \mathbf{x}_{t}^{v}\right\rangle, \\
\mathbb{E}\left\{\left\langle H x_{T}^{v}, x_{T}^{v}\right\rangle+\left\langle\bar{H} \mathbb{E} x_{T}^{v}, \mathbb{E} x_{T}^{v}\right\rangle\right\} & =\mathbb{E}\left\langle\mathbf{H} \mathbf{x}_{T}^{v}, \mathbf{x}_{T}^{v}\right\rangle, \\
\mathbb{E}\left\langle M y_{0}^{v}, y_{0}^{v}\right\rangle=\mathbb{E}\left\langle\mathbf{M} \mathbf{y}_{0}^{v}, \mathbf{y}_{0}^{v}\right\rangle, \mathbb{E}\left\langle B_{t} v_{t}, v_{t}\right\rangle & =\mathbb{E}\left\langle\mathbf{B}_{t} \mathbf{v}_{t}, \mathbf{v}_{t}\right\rangle .
\end{aligned}
$$

Then the cost functional is rewritten as

$$
\begin{aligned}
J[v]= & \mathbb{E}\left[\int_{0}^{T}\left(\left\langle\mathbf{A}_{t} \mathbf{x}_{t}^{v}, \mathbf{x}_{t}^{v}\right\rangle+\left\langle\mathbf{B}_{t} \mathbf{v}_{t}, \mathbf{v}_{t}\right\rangle\right) d t\right. \\
& \left.+\left\langle\mathbf{H} \mathbf{x}_{T}^{v}, \mathbf{x}_{T}^{v}\right\rangle+\left\langle\mathbf{M} \mathbf{y}_{0}^{v}, \mathbf{y}_{0}^{v}\right\rangle\right] .
\end{aligned}
$$

Note that (14) together with (12) and (13) forms a standard-looking LQ problem for FBSDE with noisy observation. However, the BSDE in (12) is not a standard form due to the irreversibility of $\check{c}$. Moreover, the control domain has to satisfy some extra constraint conditions according to the form of the control $\mathbf{v}$. This implies that Example (MFLQ) cannot be reduced to a standard LQ problem for FBSDE, hence it cannot be immediately solved by the standard LQ theory for FBSDE.

In the end of this section, we give a preliminary result, which shows that the desired optimality condition can be derived by minimizing $J[v]$ over $\mathcal{U}_{a d}^{0}$.

\section{Theorem 2.1.}

$$
\inf _{v^{\prime} \in \mathscr{U}_{a d}} J\left[v^{\prime}\right]=\inf _{v \in \mathscr{U}_{a d}^{0}} J[v]
$$

The proof can be found in Appendix.

\section{Optimal solution of Problem (MFC)}

For any $v, v_{j} \in \mathscr{U}_{a d}$, let $\left(x^{v}, y^{v}, z^{v}, \tilde{z}^{v}\right)$ and $\left(x^{v_{j}}, y^{v_{j}}, z^{v_{j}}\right.$, $\tilde{z}^{v_{j}}$ ) be the solutions of (7) corresponding to $v$ and $v_{j}$, $j=1,2, \cdots$. For simplicity, we set

$$
\begin{aligned}
& \left(\Theta_{t}^{\lambda}\right)=\left(t, x_{t}^{\lambda}, \mathbb{E} x_{t}^{\lambda}, \lambda_{t}\right), \quad\left(\Xi_{t}^{\lambda}\right)=\left(x_{t}^{\lambda}, \mathbb{E} x_{t}^{\lambda}\right), \\
& \left(\Pi_{t}^{\lambda}\right)=\left(x_{t}^{\lambda}, y_{t}^{\lambda}, z_{t}^{\lambda}, \tilde{z}_{t}^{\lambda}, \mathbb{E} x_{t}^{\lambda}, \mathbb{E} y_{t}^{\lambda}, \mathbb{E} z_{t}^{\lambda}, \mathbb{E} \tilde{z}_{t}^{\lambda}\right)
\end{aligned}
$$

with $\lambda=v, u, v_{j}, j=1,2, \cdots$.

\subsection{Optimality conditions}

According to Theorem 2.1 above, the optimality conditions can be derived by minimizing $J[v]$ over $\mathscr{U}_{a d}^{0}$ subject to (7) and (8). We remind reader again that these results are different from the existing literature, mainly due to some new features of Problem (MFC). For example, the state is governed by a mean-field FBSDE, and is partially observed via a noisy process.

Theorem 3.1. Assume that $u$ is an optimal control for Problem (MFC). Then the mean-field FBSDE

$$
\left\{\begin{aligned}
d k_{t}= & \left(\beta_{t}^{\top} k_{t}+\bar{\beta}_{t}^{\top} \mathbb{E} k_{t}\right) d t+\left(\gamma_{t}^{\top} k_{t}+\bar{\gamma}_{t}^{\top} \mathbb{E} k_{t}\right) d w_{t} \\
& +\left(\tilde{\gamma}_{t}^{\top} k_{t}+\tilde{\tilde{\gamma}}_{t}^{\top} \mathbb{E} k_{t}\right) d \tilde{w}_{t}, \\
-d p_{t}= & {\left[a_{t}^{\top} p_{t}+l_{x}^{\top}\left(\Theta_{t}^{u}\right)+\mathbb{E}\left(\bar{a}_{t}^{\top} p_{t}+l_{\bar{x}}^{\top}\left(\Theta_{t}^{u}\right)\right)-\alpha_{t}^{\top} k_{t}\right.} \\
& \left.-\bar{\alpha}_{t}^{\top} \mathbb{E} k_{t}\right] d t-q_{t} d w_{t}-\tilde{q}_{t} d \tilde{w}_{t}, \\
k_{0}= & -\varphi_{y}^{\top}\left(y_{0}^{u}\right), \\
p_{T}= & \phi_{x}^{\top}\left(\Xi_{T}^{u}\right)+\mathbb{E} \phi_{\bar{x}}^{\top}\left(\Xi_{T}^{u}\right)-\rho^{\top} k_{T}-\bar{\rho}^{\top} \mathbb{E} k_{T}
\end{aligned}\right.
$$

has a unique solution $(k, p, q, \tilde{q})$ in the space $\mathscr{L}_{\mathscr{F}}^{2}(0, T$; $\left.\mathbb{R}^{m+n+n \times r+n \times \tilde{r}}\right)$ such that

$$
\mathbb{E}\left[H_{v}\left(t, \Pi_{t}^{u}, u_{t} ; k_{t}, p_{t}, q_{t}\right)\left(\nu-u_{t}\right) \mid \mathscr{F}_{t}^{Y^{u}}\right] \geq 0
$$

for any $\nu \in U$, where the Hamiltonian function $H$ is defined by

$$
\begin{aligned}
& H(t, x, y, z, \tilde{z}, \bar{x}, \bar{y}, \bar{z}, \overline{\tilde{z}}, v ; k, p, q) \\
= & \left\langle a_{t} x+\bar{a}_{t} \bar{x}+b(t, v), p\right\rangle+\left\langle c_{t}, q\right\rangle+l(t, x, \bar{x}, v) \\
& -\left\langle\alpha_{t} x+\bar{\alpha}_{t} \bar{x}+\beta_{t} y+\bar{\beta}_{t} \bar{y}+\gamma_{t} z+\bar{\gamma}_{t} \bar{z}+\tilde{\gamma}_{t} \tilde{z}\right. \\
& \left.+\overline{\tilde{\gamma}}_{t} \overline{\tilde{z}}+\psi(t, v), k\right\rangle .
\end{aligned}
$$

Proof. If $u$ is an optimal control for Problem (MFC), Theorem 2.1 implies that $J[u]=\inf _{v \in \mathscr{U}_{a d}^{0}} J[v]$. For any $v \in \mathscr{U}_{a d}^{0}$, let $\left(x^{u+\varepsilon v}, y^{u+\varepsilon v}, z^{u+\varepsilon v}, \tilde{z}^{u+\varepsilon v}\right)$ be the solution of (7) corresponding to $u+\varepsilon v, 0<\varepsilon<1$. Introduce a variational equation

$$
\left\{\begin{aligned}
\dot{x}_{1, t}^{u}= & a_{t} x_{1, t}^{u}+\bar{a}_{t} \mathbb{E} x_{1, t}^{u}+b_{v}\left(t, u_{t}\right) v_{t}, \\
-d y_{1, t}^{u}= & \left(\alpha_{t} x_{1, t}^{u}+\bar{\alpha}_{t} \mathbb{E} x_{1, t}^{u}+\beta_{t} y_{1, t}^{u}+\bar{\beta}_{t} \mathbb{E} y_{1, t}^{u}\right. \\
& +\gamma_{t} z_{1, t}^{u}+\bar{\gamma}_{t} \mathbb{E} z_{1, t}^{u}+\tilde{\gamma}_{t} \tilde{z}_{1, t}^{u}+\overline{\tilde{\gamma}}_{t} \mathbb{E} \tilde{z}_{1, t}^{u} \\
& \left.+\psi_{v}\left(t, u_{t}\right) v_{t}\right) d t-z_{1, t}^{u} d w_{t}-\tilde{z}_{1, t}^{u} d \tilde{w}_{t}, \\
x_{1,0}^{u}= & 0, \quad y_{1, T}^{u}=\rho x_{1, T}^{u}+\bar{\rho} \mathbb{E} x_{1, T}^{u},
\end{aligned}\right.
$$

which admits a unique solution $\left(x_{1}^{u}, y_{1}^{u}, z_{1}^{u}, \tilde{z}_{1}^{u}\right) \in$ $\mathscr{L}_{\mathscr{F}}^{2}\left(0, T ; \mathbb{R}^{n+m+m \times r+m \times \tilde{r}}\right)$. It follows from Taylor's expansion, Hölder's inequality and the techniques applied 
in Lemma A.1 (see Appendix) that

$$
\lim _{\varepsilon \rightarrow 0} \mathbb{E} \sup _{0 \leq t \leq T}\left|\frac{x_{t}^{u+\varepsilon v}-x_{t}^{u}}{\varepsilon}-x_{1, t}^{u}\right|^{2}=0,
$$

and then,

$$
\lim _{\varepsilon \rightarrow 0} \mathbb{E} \sup _{0 \leq t \leq T}\left|\frac{y_{t}^{u+\varepsilon v}-y_{t}^{u}}{\varepsilon}-y_{1, t}^{u}\right|^{2}=0 .
$$

Combining the limits with the optimality of $u$ using the first variation of $J[v]$, we have

$$
\begin{aligned}
0 \leq & \lim _{\varepsilon \rightarrow 0} \frac{J[u+\varepsilon v]-J[u]}{\varepsilon} \\
= & \mathbb{E} \int_{0}^{T}\left(l_{x}^{\top}\left(\Theta_{t}^{u}\right) x_{1, t}^{u}+l_{\bar{x}}^{\top}\left(\Theta_{t}^{u}\right) \mathbb{E} x_{1, t}^{u}+l_{v}^{\top}\left(\Theta_{t}^{u}\right) v_{t}\right) d t \\
& +\mathbb{E}\left(\phi_{x}^{\top}\left(\Xi_{T}^{u}\right) x_{1, T}^{u}+\phi_{\bar{x}}^{\top}\left(\Xi_{T}^{u}\right) \mathbb{E} x_{1, T}^{u}+\varphi_{y}^{\top}\left(y_{0}^{u}\right) y_{1,0}^{u}\right) .
\end{aligned}
$$

On the other hand, once $\left(x^{u}, y^{u}, z^{u}, \tilde{z}^{u}\right)$ is determined by $(7)$, there is a unique solution $(k, p, q, \tilde{q})$, in the space $\mathscr{L}_{\mathscr{F}}^{2}\left(0, T ; \mathbb{R}^{m+n+n \times r+n \times \tilde{r}}\right)$, to (15). Using Itô's formula to $\left\langle x_{1}^{u}, p\right\rangle+\left\langle y_{1}^{u}, k\right\rangle$ and inserting it into (17), we get

$$
\mathbb{E} \int_{0}^{T}\left(p_{t}^{\top} b_{v}\left(t, u_{t}\right)-k_{t}^{\top} \psi_{v}\left(t, u_{t}\right)+l_{v}^{\top}\left(\Theta_{t}^{u}\right)\right) v_{t} d t \geq 0 .
$$

Due to $u \in \mathscr{U}_{a d}^{0}$ and the arbitrariness of $v_{t}$, we deduce

$$
\begin{aligned}
& \mathbb{E}\left[\left(p_{t}^{\top} b_{v}\left(t, u_{t}\right)-k_{t}^{\top} \psi_{v}\left(t, u_{t}\right)+l_{v}^{\top}\left(\Theta_{t}^{u}\right)\right)\left(\nu-u_{t}\right) \mid \mathscr{F}_{t}^{Y^{0}}\right] \\
= & \mathbb{E}\left[H_{v}\left(t, \Pi_{t}^{u}, u_{t} ; k_{t}, p_{t}, q_{t}\right)\left(\nu-u_{t}\right) \mid \mathscr{F}_{t}^{Y^{0}}\right] \geq 0,
\end{aligned}
$$

for any $\nu \in U$. Since $u \in \mathscr{U}_{a d}$, it follows from Proposition 2.1 that $\mathscr{F}_{t}^{Y^{u}}=\mathscr{F}_{t}^{Y^{0}}$. Then the result is derived. The proof is complete.

Theorem 3.2. Assume that for any $(t, x, y, z, \tilde{z}, \bar{x}, \bar{y}, \bar{z}, \bar{z}$, $v) \in[0, T] \times \mathbb{R}^{n+m+m \times r+m \times \tilde{r}+n+m+m \times r+m \times \tilde{r}} \times U$, $(x, \bar{x}, v) \mapsto l(t, x, \bar{x}, v),(x, \bar{x}) \mapsto \phi(x, \bar{x})$ and $y \mapsto$ $\varphi(y)$ are convex. Assume that $b\left(t, v_{t}\right)=\tilde{b}_{t} v_{t}+$ $\bar{b}_{t}, \psi\left(t, v_{t}\right)=\tilde{\psi}_{t} v_{t}+\bar{\psi}_{t}$, where $\tilde{b} \in \mathscr{L}^{\infty}\left(0, T ; \mathbb{R}^{n \times k}\right)$, $\bar{b} \in \mathscr{L}^{\infty}\left(0, T ; \mathbb{R}^{n}\right), \tilde{\psi} \in \mathscr{L}^{\infty}\left(0, T ; \mathbb{R}^{m \times k}\right), \bar{\psi} \in$ $\mathscr{L}^{\infty}\left(0, T ; \mathbb{R}^{m}\right)$. Let $u \in \mathscr{U}_{\text {ad }}$ and

$$
\begin{aligned}
& \mathbb{E}\left[H\left(t, \Pi_{t}^{u}, u_{t} ; k_{t}, p_{t}, q_{t}\right) \mid \mathscr{F}_{t}^{Y^{u}}\right] \\
= & \inf _{v \in U} \mathbb{E}\left[H\left(t, \Pi_{t}^{u}, v ; k_{t}, p_{t}, q_{t}\right) \mid \mathscr{F}_{t}^{Y^{u}}\right],
\end{aligned}
$$

where $\left(x^{u}, y^{u}, z^{u}, \tilde{z}^{u}\right) \in \mathscr{L}_{\mathscr{F}}^{2}\left(0, T ; \mathbb{R}^{n+m+m \times r+m \times \tilde{r}}\right)$ is the solution to (7) under the admissible control $u$, and $(k, p, q, \tilde{q}) \in \mathscr{L}_{\mathscr{F}}^{2}\left(0, T ; \mathbb{R}^{m+n+n \times r+n \times \tilde{r}}\right)$ is the solution to (15). Then u is an optimal control of Problem (MFC).

Proof. For any $v \in \mathscr{U}_{a d}$, we write

$$
J[v]-J[u]=I_{1}+I_{2}+I_{3}
$$

with

$$
\begin{aligned}
& I_{1}=\mathbb{E} \int_{0}^{T}\left(l\left(\Theta_{t}^{v}\right)-l\left(\Theta_{t}^{u}\right)\right) d t, I_{2}=\mathbb{E}\left[\phi\left(\Xi_{T}^{v}\right)-\phi\left(\Xi_{T}^{u}\right)\right], \\
& I_{3}=\mathbb{E}\left[\varphi\left(y_{0}^{v}\right)-\varphi\left(y_{0}^{u}\right)\right] .
\end{aligned}
$$

By virtue of the convexity of $\phi$ and applying Itô's formula to $\left\langle p, x^{v}-x^{u}\right\rangle$, we have

$$
\begin{gathered}
I_{2} \geq \mathbb{E}\left\langle p_{T}, x_{T}^{v}-x_{T}^{u}\right\rangle+\mathbb{E}\left\langle\rho^{\top} k_{T}+\mathbb{E}\left(\bar{\rho}^{\top} k_{T}\right), x_{T}^{v}-x_{T}^{u}\right\rangle \\
=-\mathbb{E} \int_{0}^{T}\left\langle l_{x}^{\top}\left(\Theta_{t}^{u}\right)+\mathbb{E} l_{\bar{x}}^{\top}\left(\Theta_{t}^{u}\right)-\alpha_{t}^{\top} k_{t}-\mathbb{E}\left(\bar{\alpha}_{t}^{\top} k_{t}\right),\right. \\
\left.x_{t}^{v}-x_{t}^{u}\right\rangle d t+\mathbb{E} \int_{0}^{T}\left\langle p_{t}, \tilde{b}_{t}\left(v_{t}-u_{t}\right)\right\rangle d t \\
+\mathbb{E}\left\langle\rho^{\top} k_{T}+\mathbb{E}\left(\bar{\rho}^{\top} k_{T}\right), x_{T}^{v}-x_{T}^{u}\right\rangle .
\end{gathered}
$$

Similarly, applying Itô's formula to $\left\langle k, y^{v}-y^{u}\right\rangle$ and the convexity of $\varphi$, we derive

$$
\begin{gathered}
I_{3} \geq-\mathbb{E}\left\langle k_{T}, \rho\left(x_{T}^{v}-x_{T}^{u}\right)+\bar{\rho} \mathbb{E}\left(x_{T}^{v}-x_{T}^{u}\right)\right\rangle \\
-\mathbb{E} \int_{0}^{T}\left\langle k_{t}, \alpha_{t}\left(x_{t}^{v}-x_{t}^{u}\right)+\bar{\alpha}_{t} \mathbb{E}\left(x_{t}^{v}-x_{t}^{u}\right)\right. \\
\left.+\tilde{\psi}_{t}\left(v_{t}-u_{t}\right)\right\rangle d t .
\end{gathered}
$$

It is easy to see from (19), (20), (21) and the convexity of $l$ that

$$
\begin{aligned}
& J[v]-J[u] \\
\geq & \mathbb{E} \int_{0}^{T} H_{v}\left(t, \Pi_{t}^{u}, u_{t} ; k_{t}, p_{t}, q_{t}\right)\left(v_{t}-u_{t}\right) d t \\
= & \mathbb{E} \int_{0}^{T} \mathbb{E}\left[H_{v}\left(t, \Pi_{t}^{u}, u_{t} ; k_{t}, p_{t}, q_{t}\right)\left(v_{t}-u_{t}\right) \mid \mathscr{F}_{t} Y^{0}\right] d t .
\end{aligned}
$$

Further, using Theorem 2.1 and (18), we get

$$
\begin{aligned}
& \mathbb{E}\left[H_{v}\left(t, \Pi_{t}^{u}, u_{t} ; k_{t}, p_{t}, q_{t}\right)\left(v_{t}-u_{t}\right) \mid \mathscr{F}_{t}^{Y^{u}}\right] \\
= & \frac{\partial}{\partial v} \mathbb{E}\left[H\left(t, \Pi_{t}^{u}, u_{t} ; k_{t}, p_{t}, q_{t}\right) \mid \mathscr{F}_{t}^{Y^{u}}\right]\left(v_{t}-u_{t}\right) \geq 0 .
\end{aligned}
$$

Then this implies the desired result.

\subsection{Optimal filters}

The minimum condition (16) shows that we need to analyze the optimal filters of (7) and (15) depend- 
ing on $\mathscr{F}_{t}^{Y^{v}}$ in order to compute $u$. For this, for any $v \in \mathscr{U}_{a d}$ we denote by $\hat{\iota}_{t}=\mathbb{E}\left[\iota_{t} \mid \mathscr{F}_{t}^{Y^{v}}\right]$ with $\iota_{t}=x_{t}^{0}, x_{t}^{v}, y_{t}^{v}, z_{t}^{v}, \tilde{z}_{t}^{v}, y_{t}^{v}\left(x_{t}^{v}\right)^{\top}$ and $\hat{\kappa}_{t}=\mathbb{E}\left[\kappa_{t} \mid \mathscr{F}_{t} Y^{u}\right]$ with $\kappa_{t}=k_{t}, p_{t}, p_{t}\left(x_{t}^{u}\right)^{\top}, k_{t}\left(x_{t}^{u}\right)^{\top}, \tilde{q}_{t}$ the optimal filters of $\iota_{t}$ and $\kappa_{t}$, respectively. Moreover, we denote by $\Sigma_{t}=\mathbb{E}\left[\left(x_{t}^{v}-\hat{x}_{t}^{v}\right)\left(x_{t}^{v}-\hat{x}_{t}^{v}\right)^{\top}\right]$ the mean square error of $\hat{x}_{t}^{v}, v \in \mathscr{U}_{a d}$.

Using Theorem 12.7 in Liptser and Shiryayev [30] and Theorem 3.1 in Wang et al. [42], we derive (22) and (23), respectively.

Theorem 3.3. For any $v \in \mathscr{U}_{a d}$, the optimal filters $\left(\hat{x}_{t}^{v}, \hat{y}_{t}^{v}\right)$ and $\left(\hat{k}_{t}^{v}, \hat{p}_{t}^{v}\right)$ of the solutions $\left(x_{t}^{v}, y_{t}^{v}\right)$ and $\left(k_{t}, p_{t}\right)$ to (7) and (15) with respect to $\mathscr{F}_{t}^{Y^{v}}$ and $\mathscr{F}_{t}^{Y^{u}}$ satisfy

$$
\left\{\begin{aligned}
d \hat{x}_{t}^{v}= & \left(a_{t} \hat{x}_{t}^{v}+\bar{a}_{t} \mathbb{E} x_{t}^{v}+b\left(t, v_{t}\right)\right) d t \\
& +\Sigma_{t} f_{t}^{\top}\left(h_{t}^{-1}\right)^{\top} d \bar{w}_{t}, \\
-d \hat{y}_{t}^{v}= & \left(\alpha_{t} \hat{x}_{t}^{v}+\bar{\alpha}_{t} \mathbb{E} x_{t}^{v}+\beta_{t} \hat{y}_{t}^{v}+\bar{\beta}_{t} \mathbb{E} y_{t}^{v}+\gamma_{t} \hat{z}_{t}^{v}\right. \\
& \left.+\bar{\gamma}_{t} \mathbb{E} z_{t}^{v}+\tilde{\gamma}_{t} \hat{\tilde{z}}_{t}^{v}+\overline{\tilde{\gamma}}_{t} \mathbb{E} \tilde{z}_{t}^{v}+\psi\left(t, v_{t}\right)\right) d t \\
& -\hat{\tilde{Z}}_{t}^{v} d \bar{w}_{t}, \\
\hat{x}_{0}^{v}= & \mu_{0}, \quad \hat{y}_{T}^{v}=\rho \hat{x}_{T}^{v}+\bar{\rho} \mathbb{E} x_{T}^{v},
\end{aligned}\right.
$$

and

$$
\left\{\begin{aligned}
d \hat{k}_{t}= & \left(\beta_{t}^{\top} \hat{k}_{t}+\bar{\beta}_{t}^{\top} \mathbb{E} k_{t}\right) d t+\left[\tilde{\gamma}_{t}^{\top} \hat{k}_{t}+\overline{\tilde{\gamma}}_{t}^{\top} \mathbb{E} k_{t}\right. \\
& \left.+\left(\widehat{k_{t}\left(x_{t}^{u}\right)^{\top}}-\hat{k}_{t}\left(\hat{x}_{t}^{u}\right)^{\top}\right) f_{t}^{\top}\left(h_{t}^{-1}\right)^{\top}\right] d \bar{w}_{t}, \\
-d \hat{p}_{t}= & \left\{a_{t}^{\top} \hat{p}_{t}+\mathbb{E}\left[l_{x}^{\top}\left(\Theta_{t}^{u}\right) \mid \mathscr{F}_{t}^{Y^{u}}\right]-\alpha_{t}^{\top} \hat{k}_{t}-\bar{\alpha}_{t}^{\top} \mathbb{E} k_{t}\right. \\
& \left.+\mathbb{E}\left(\bar{a}_{t}^{\top} p_{t}+l_{\bar{x}}^{\top}\left(\Theta_{t}^{u}\right)\right)\right\} d t-\widehat{\tilde{Q}}_{t} d \bar{w}_{t}, \\
\hat{k}_{0}= & -\varphi_{y}^{\top}\left(\hat{y}_{0}^{u}\right), \\
\hat{p}_{T}= & \mathbb{E}\left[\phi_{x}^{\top}\left(\Xi_{T}^{u}\right) \mid \mathscr{F}_{T}^{Y^{u}}\right]+\mathbb{E} \phi_{\bar{x}}^{\top}\left(\Xi_{T}^{u}\right) \\
& -\rho^{\top} \hat{k}_{T}-\bar{\rho}^{\top} \mathbb{E} k_{T},
\end{aligned}\right.
$$

respectively, where $\Sigma$ is the unique solution of

$$
\left\{\begin{array}{l}
\dot{\Sigma}_{t}-a_{t} \Sigma_{t}-\Sigma_{t} a_{t}^{\top}+\Sigma_{t} f_{t}^{\top}\left(h_{t}^{-1}\right)^{\top} h_{t}^{-1} f_{t} \Sigma_{t} \\
-c_{t} c_{t}^{\top}=0, \\
\Sigma_{0}=\sigma_{0},
\end{array}\right.
$$

$\bar{w}_{t}=\int_{0}^{t} h_{s}^{-1}\left[d Y_{s}^{0}-\left(f_{s} \hat{x}_{s}^{0}+\bar{f}_{s} \mathbb{E} x_{s}^{0}\right) d s\right]$

is a standard Brownian motion with value in $\mathbb{R}^{\tilde{r}}$, and

$$
\widehat{\tilde{Z}}_{t}=\hat{\tilde{z}}_{t}+\left(y_{t}^{v\left(x_{t}^{v}\right)^{\top}}-\hat{y}_{t}^{v}\left(\hat{x}_{t}^{v}\right)^{\top}\right) f_{t}^{\top}\left(h_{t}^{-1}\right)^{\top},
$$

$$
\widehat{\tilde{Q}}_{t}=\hat{\tilde{q}}_{t}+\left(\widehat{p_{t}\left(x_{t}^{u}\right)^{\top}}-\hat{p}_{t}\left(\hat{x}_{t}^{u}\right)^{\top}\right) f_{t}^{\top}\left(h_{t}^{-1}\right)^{\top} .
$$

We emphasize that (22) and (23) are two forwardbackward optimal filters. It shows that the difference between Theorem 3.3 and the classical filtering literature, say, Bensoussan [2,3], Liptser and Shiryayev [30].

\section{An LQ case of Problem (MFC)}

We still adopt the notations and the assumptions introduced in Sections 2 and 3 unless noted otherwise.

Problem (MFLQ). Minimize

$$
\begin{aligned}
J[v]= & \frac{1}{2} \mathbb{E}\left\{\int _ { 0 } ^ { T } \left[\left\langle A_{t} x_{t}^{v}, x_{t}^{v}\right\rangle+\left\langle\bar{A}_{t} \mathbb{E} x_{t}^{v}, \mathbb{E} x_{t}^{v}\right\rangle\right.\right. \\
& +\left\langle B_{t} v_{t}, v_{t}\right\rangle+2\left\langle D_{t} x_{t}^{v}, v_{t}\right\rangle+2\left\langle\bar{D}_{t} \mathbb{E} x_{t}^{v}, v_{t}\right\rangle \\
& \left.+2\left\langle\tilde{F}_{t}, x_{t}^{v}\right\rangle+2\left\langle\tilde{\bar{F}}_{t}, \mathbb{E} x_{t}^{v}\right\rangle+2\left\langle\tilde{G}_{t}, v_{t}\right\rangle\right] d t \\
& +\left\langle H x_{T}^{v}, x_{T}^{v}\right\rangle+\left\langle\bar{H} \mathbb{E} x_{T}^{v}, \mathbb{E} x_{T}^{v}\right\rangle+2\left\langle\tilde{L}, x_{T}^{v}\right\rangle \\
& \left.+2\left\langle\tilde{\bar{L}}, \mathbb{E} x_{T}^{v}\right\rangle+\left\langle M y_{0}^{v}, y_{0}^{v}\right\rangle+2\left\langle N, y_{0}^{v}\right\rangle\right\}
\end{aligned}
$$

over $\mathscr{U}_{a d}$ with the control domain $U=\mathbb{R}^{k}$, subject to the state equation

$$
\left\{\begin{aligned}
d x_{t}^{v}= & \left(a_{t} x_{t}^{v}+\bar{a}_{t} \mathbb{E} x_{t}^{v}+b_{t} v_{t}+\bar{b}_{t}\right) d t+c_{t} d w_{t}, \\
-d y_{t}^{v}= & \left(\alpha_{t} x_{t}^{v}+\bar{\alpha}_{t} \mathbb{E} x_{t}^{v}+\beta_{t} y_{t}^{v}+\bar{\beta}_{t} \mathbb{E} y_{t}^{v}+\gamma_{t} z_{t}^{v}\right. \\
& \left.+\bar{\gamma}_{t} \mathbb{E} z_{t}^{v}+\tilde{\gamma}_{t} \tilde{z}_{t}^{v}+\tilde{\tilde{\gamma}}_{t} \mathbb{E} \tilde{z}_{t}^{v}+\psi_{t} v_{t}+\bar{\psi}_{t}\right) d t \\
& -z_{t}^{v} d w_{t}-\tilde{z}_{t}^{v} d \tilde{w}_{t}, \\
x_{0}^{v}= & \xi, \quad y_{T}^{v}=\rho x_{T}^{v}+\bar{\rho} \mathbb{E} x_{T}^{v},
\end{aligned}\right.
$$

and the observation equation

$$
\left\{\begin{aligned}
d Y_{t}^{v} & =\left(f_{t} x_{t}^{v}+\bar{f}_{t} \mathbb{E} x_{t}^{v}+g_{t}\right) d t+h_{t} d \tilde{w}_{t}, \\
Y_{0}^{v} & =0
\end{aligned}\right.
$$

where $A, \bar{A} \in \mathscr{L}^{\infty}\left(0, T ; S^{n}\right), B \in \mathscr{L}^{\infty}\left(0, T ; S^{k}\right)$, $H, \bar{H} \in S^{n}, A, H, M \geq 0, B>0, A+\bar{A}, H+\bar{H} \geq 0$, $D, \bar{D} \in \mathscr{L}^{\infty}\left(0, T ; \mathbb{R}^{k \times n}\right), \quad \tilde{F}, \tilde{\bar{F}} \in \mathscr{L}^{\infty}\left(0, T ; \mathbb{R}^{n}\right)$, $\tilde{G} \in \mathscr{L}^{\infty}\left(0, T ; \mathbb{R}^{k}\right), L, \bar{L} \in \mathbb{R}^{n}, N \in \mathbb{R}^{m}, b \in$ $\mathscr{L}^{\infty}\left(0, T ; \mathbb{R}^{n \times k}\right), \bar{b} \in \mathscr{L}^{\infty}\left(0, T ; \mathbb{R}^{n}\right), \psi \in \mathscr{L}^{\infty}\left(0, T ; \mathbb{R}^{m \times k}\right)$, $\bar{\psi} \in \mathscr{L}^{\infty}\left(0, T ; \mathbb{R}^{m}\right)$, and $g \in \mathscr{L}^{\infty}\left(0, T ; \mathbb{R}^{\tilde{r}}\right)$.

Note that we do not assume the positive semidefiniteness of $\bar{A}$ and $\bar{H}$ in Problem (MFLQ). Then (26) covers the performance functional of Problem (AL) as a special case.

Proposition 4.1. If $u$ is an optimal control for Problem 
(MFLQ), then

$$
\begin{aligned}
u_{t}= & -B_{t}^{-1}\left(b_{t}^{\top} \mathbb{E}\left[p_{t} \mid \mathscr{F}_{t}^{Y^{u}}\right]-\psi_{t}^{\top} \mathbb{E}\left[k_{t} \mid \mathscr{F}_{t}^{Y^{u}}\right]\right. \\
& \left.+D_{t} \mathbb{E}\left[x_{t}^{u} \mid \mathscr{F}_{t}^{Y^{u}}\right]+\bar{D}_{t} \mathbb{E} x_{t}^{u}+\tilde{G}_{t}\right),
\end{aligned}
$$

where $(k, p)$ is the solution of the adjoint equation

$$
\left\{\begin{aligned}
d k_{t}= & \left(\beta_{t}^{\top} k_{t}+\bar{\beta}_{t}^{\top} \mathbb{E} k_{t}\right) d t+\left(\gamma_{t}^{\top} k_{t}+\bar{\gamma}_{t}^{\top} \mathbb{E} k_{t}\right) d w_{t} \\
& +\left(\tilde{\gamma}_{t}^{\top} k_{t}+\overline{\tilde{\gamma}}_{t}^{\top} \mathbb{E} k_{t}\right) d \tilde{w}_{t}, \\
-d p_{t}= & {\left[a_{t}^{\top} p_{t}-\alpha_{t}^{\top} k_{t}+A_{t} x_{t}^{u}+D_{t}^{\top} u_{t}+F_{t}\right.} \\
& \left.+\mathbb{E}\left(\bar{a}_{t}^{\top} p_{t}-\bar{\alpha}_{t}^{\top} k_{t}+\bar{A}_{t} x_{t}^{u}+\bar{D}_{t}^{\top} u_{t}+\bar{F}_{t}\right)\right] d t \\
& -q_{t} d w_{t}-\tilde{q}_{t} d \tilde{w}_{t}, \\
k_{0}= & -M y_{0}^{u}-N, \\
p_{T}= & H x_{T}^{u}+\bar{H} \mathbb{E} x_{T}^{u}+\tilde{L}+\tilde{\bar{L}}-\rho^{\top} k_{T}-\bar{\rho}^{\top} \mathbb{E} k_{T},
\end{aligned}\right.
$$

together with the state equation

$$
\left\{\begin{aligned}
d x_{t}^{u}= & \left(a_{t} x_{t}^{u}+\bar{a}_{t} \mathbb{E} x_{t}^{u}+b_{t} u_{t}+\bar{b}_{t}\right) d t+c_{t} d w_{t}, \\
-d y_{t}^{u}= & \left(\alpha_{t} x_{t}^{u}+\bar{\alpha}_{t} \mathbb{E} x_{t}^{u}+\beta_{t} y_{t}^{u}+\bar{\beta}_{t} \mathbb{E} y_{t}^{u}+\gamma_{t} z_{t}^{u}\right. \\
& \left.+\bar{\gamma}_{t} \mathbb{E} z_{t}^{u}+\tilde{\gamma}_{t} \tilde{z}_{t}^{u}+\overline{\tilde{\gamma}}_{t} \mathbb{E} \tilde{z}_{t}^{u}+\psi_{t} u_{t}+\bar{\psi}_{t}\right) d t \\
& -z_{t}^{u} d w_{t}-\tilde{z}_{t}^{u} d \tilde{w}_{t} \\
x_{0}^{u}= & \xi, \quad y_{T}^{u}=\rho x_{T}^{u}+\bar{\rho} \mathbb{E} x_{T}^{u} .
\end{aligned}\right.
$$

Proof. With the above data, the Hamiltonian function is

$$
\begin{aligned}
& H(t, x, y, z, \tilde{z}, \bar{x}, \bar{y}, \bar{z}, \overline{\tilde{z}}, v ; k, p, q) \\
= & \left\langle a_{t} x+\bar{a}_{t} \bar{x}+b_{t} v+\bar{b}_{t}, p\right\rangle+\left\langle c_{t}, q\right\rangle-\left\langle\alpha_{t} x+\bar{\alpha}_{t} \bar{x}+\beta_{t} y\right. \\
& \left.+\bar{\beta}_{t} \bar{y}+\gamma_{t} z+\bar{\gamma}_{t} \bar{z}+\tilde{\gamma}_{t} \tilde{z}+\overline{\tilde{\gamma}}_{t} \overline{\tilde{z}}+\psi_{t} v+\bar{\psi}_{t}, k\right\rangle \\
& +\frac{1}{2}\left[\left\langle A_{t} x, x\right\rangle+\left\langle\bar{A}_{t} \bar{x}, \bar{x}\right\rangle+\left\langle B_{t} v, v\right\rangle+2\left\langle D_{t} x, v\right\rangle\right. \\
& \left.+2\left\langle\bar{D}_{t} \bar{x}, v\right\rangle+2\left\langle\tilde{F}_{t}, x\right\rangle+2\left\langle\tilde{\bar{F}}_{t}, \bar{x}\right\rangle+2\left\langle\tilde{G}_{t}, v\right\rangle\right],
\end{aligned}
$$

where $(k, p, q, \tilde{q})$ is determined by (29) together with (30). If $u(\cdot)$ is optimal, it follows from Theorem 3.1 that

$$
\begin{aligned}
u_{t}= & -B_{t}^{-1}\left(b_{t}^{\top} \mathbb{E}\left[p_{t} \mid \mathscr{F}_{t}^{Y^{u}}\right]-\psi_{t}^{\top} \mathbb{E}\left[k_{t} \mid \mathscr{F}_{t}^{Y^{u}}\right]\right. \\
& \left.+D_{t} \mathbb{E}\left[x_{t}^{u} \mid \mathscr{F}_{t}^{Y^{u}}\right]+\bar{D}_{t} \mathbb{E} x_{t}^{u}+\tilde{G}_{t}\right),
\end{aligned}
$$

where $\left(x^{u}, k, p\right)$ is the solution of (30) with (29). Then the proof is complete.

Note that one more explicit optimal control $u$ strong depends on a certain special structure of the state equation and the cost functional. Next, let us consider a particular case of Problem (MFLQ), i.e., let $M=0$ and $\bar{\beta}_{t}=\gamma_{t}=\bar{\gamma}_{t}=\tilde{\gamma}_{t}=\overline{\tilde{\gamma}}_{t}=0$ in (26) and (27), respec- tively. By Theorems 3.1, 3.2 and 3.3, an optimal feedback control is explicitly obtained. The procedure of how to solve is decomposed into five steps below. Note that such an optimal control will play a role in Problem (AL). Please refer to Section 5 below for more details.

Step 1: A reduced LQ problem.

Integrating and taking expectations on both sides of the BSDE in (27), we have

$$
\begin{aligned}
\mathbb{E} y_{t}^{v}= & \mathbb{E}\left[\chi_{t}^{T} \rho x_{T}^{v}+\chi_{t}^{T} \bar{\rho} \mathbb{E} x_{T}^{v}\right. \\
& \left.+\int_{t}^{T} \chi_{t}^{s}\left(\alpha_{s} x_{s}^{v}+\bar{\alpha}_{s} \mathbb{E} x_{s}^{v}+\psi_{s} v_{s}+\bar{\psi}_{s}\right) d s\right]
\end{aligned}
$$

with

$$
\chi_{t}^{s}=e^{\int_{t}^{s}\left(\beta_{\tau}+\bar{\beta}_{\tau}\right) d \tau}, \quad t \leq s \leq T .
$$

Plugging the equality into (26), we derive an LQ problem for mean-field SDE as follows.

Problem (MFLQ)'. Find a $v \in \mathscr{U}_{a d}$ to minimize

$$
\begin{aligned}
J[v]= & \frac{1}{2} \mathbb{E}\left\{\int _ { 0 } ^ { T } \left[\left\langle A_{t} x_{t}^{v}, x_{t}^{v}\right\rangle+\left\langle\bar{A}_{t} \mathbb{E} x_{t}^{v}, \mathbb{E} x_{t}^{v}\right\rangle\right.\right. \\
& +\left\langle B_{t} v_{t}, v_{t}\right\rangle+2\left\langle D_{t} x_{t}^{v}, v_{t}\right\rangle+2\left\langle\bar{D}_{t} \mathbb{E} x_{t}^{v}, v_{t}\right\rangle \\
& \left.+2\left\langle F_{t}, x_{t}^{v}\right\rangle+2\left\langle\bar{F}_{t}, \mathbb{E} x_{t}^{v}\right\rangle+2\left\langle G_{t}, v_{t}\right\rangle\right] d t \\
& +\left\langle H x_{T}^{v}, x_{T}^{v}\right\rangle+\left\langle\bar{H} \mathbb{E} x_{T}^{v}, \mathbb{E} x_{T}^{v}\right\rangle+2\left\langle L, x_{T}^{v}\right\rangle \\
& \left.+2\left\langle\bar{L}, \mathbb{E} x_{T}^{v}\right\rangle\right\}+J_{0}
\end{aligned}
$$

subject to

$$
\left\{\begin{aligned}
d x_{t}^{v} & =\left(a_{t} x_{t}^{v}+\bar{a}_{t} \mathbb{E} x_{t}^{v}+b_{t} v_{t}+\bar{b}_{t}\right) d t+c_{t} d w_{t} \\
x_{0}^{v} & =\xi
\end{aligned}\right.
$$

and (28) with

$$
\begin{aligned}
F & =\tilde{F}+\left(\chi_{0}^{t} \alpha_{t}\right)^{\top} N, & \bar{F} & =\tilde{\bar{F}}+\left(\chi_{0}^{t} \bar{\alpha}_{t}\right)^{\top} N, \\
L & =\tilde{L}+\left(\chi_{0}^{T} \rho\right)^{\top} N, & \bar{L} & =\tilde{\bar{L}}+\left(\chi_{0}^{T} \bar{\rho}\right)^{\top} N, \\
G & =\tilde{G}+\left(\chi_{0}^{t} \psi_{t}\right)^{\top} N, & J_{0} & =\int_{0}^{T} N^{\top} \chi_{0}^{t} \bar{\psi}_{t} d t .
\end{aligned}
$$

Step 2: Candidate optimal control.

The Hamiltonian function is

$$
\begin{aligned}
H(t, x, \bar{x}, v ; p, q)= & \left\langle a_{t} x+\bar{a}_{t} \bar{x}+b_{t} v+\bar{b}_{t}, p\right\rangle+\left\langle c_{t}, q\right\rangle \\
& +\frac{1}{2}\left[\left\langle A_{t} x, x\right\rangle+\left\langle\bar{A}_{t} \bar{x}, \bar{x}\right\rangle+\left\langle B_{t} v, v\right\rangle\right. \\
& +2\left\langle D_{t} x, v\right\rangle+2\left\langle\bar{D}_{t} \bar{x}, v\right\rangle+2\left\langle F_{t}, x\right\rangle \\
& \left.+2\left\langle\bar{F}_{t}, \bar{x}\right\rangle+2\left\langle G_{t}, v\right\rangle\right],
\end{aligned}
$$


where $(p, q)$ is determined by the Hamiltonian system

$$
\left\{\begin{aligned}
d x_{t}^{u}= & \left(a_{t} x_{t}^{u}+\bar{a}_{t} \mathbb{E} x_{t}^{u}+b_{t} u_{t}+\bar{b}_{t}\right) d t+c_{t} d w_{t}, \\
-d p_{t}= & {\left[a_{t}^{\top} p_{t}+A_{t} x_{t}^{u}+D_{t}^{\top} u_{t}+F_{t}\right.} \\
& \left.+\mathbb{E}\left(\bar{a}_{t}^{\top} p_{t}+\bar{A}_{t} x_{t}^{u}+\bar{D}_{t}^{\top} u_{t}+\bar{F}_{t}\right)\right] d t \\
& -q_{t} d w_{t}-\tilde{q}_{t} d \tilde{w}_{t} \\
x_{0}^{u}= & \xi, \quad p_{T}=H x_{T}^{u}+\bar{H} \mathbb{E} x_{T}^{u}+L+\bar{L} .
\end{aligned}\right.
$$

If $u$ is optimal, then it follows from Theorem 3.1 or Proposition 4.1 that

$$
\begin{aligned}
u_{t}= & -B_{t}^{-1}\left(b_{t}^{\top} \mathbb{E}\left[p_{t} \mid \mathscr{F}_{t}^{Y^{u}}\right]+D_{t} \mathbb{E}\left[x_{t}^{u} \mid \mathscr{F}_{t}^{Y^{u}}\right]\right. \\
& \left.+\bar{D}_{t} \mathbb{E} x_{t}^{u}+G_{t}\right) \\
= & -B_{t}^{-1}\left(b_{t}^{\top} \hat{p}_{t}+D_{t} \hat{x}_{t}^{u}+\bar{D}_{t} \mathbb{E} x_{t}^{u}+G_{t}\right) .
\end{aligned}
$$

Step 3: Feedback representation of (32).

Inserting (32) into (31) and taking expectations, we get a fully coupled forward-backward ordinary differential equation (ODE, in short)

$$
\left\{\begin{aligned}
\mathbb{E} \dot{x}_{t}^{u}= & {\left[a_{t}+\bar{a}_{t}-b_{t} B_{t}^{-1}\left(D_{t}+\bar{D}_{t}\right)\right] \mathbb{E} x_{t}^{u} } \\
& -b_{t} B_{t}^{-1} b_{t}^{\top} \mathbb{E} p_{t}-b_{t} B_{t}^{-1} G_{t}+\bar{b}_{t}, \\
\dot{\mathbb{E} p_{t}=} & -\left[A_{t}+\bar{A}_{t}-\left(D_{t}+\bar{D}_{t}\right)^{\top} B_{t}^{-1}\left(D_{t}+\bar{D}_{t}\right)\right] \mathbb{E} x_{t}^{u} \\
& -\left[\left(a_{t}+\bar{a}_{t}\right)^{\top}-\left(D_{t}+\bar{D}_{t}\right)^{\top} B_{t}^{-1} b_{t}^{\top}\right] \mathbb{E} p_{t} \\
& +\left(D_{t}+\bar{D}_{t}\right)^{\top} B_{t}^{-1} G_{t}-F_{t}-\bar{F}_{t}, \\
\mathbb{E} x_{0}^{u}= & \mu_{0}, \quad \mathbb{E} p_{T}=(H+\bar{H}) \mathbb{E} x_{T}^{u}+L+\bar{L} .
\end{aligned}\right.
$$

According to Theorem 2.6 in Peng and $\mathrm{Wu}[36],(33)$ has a unique solution $\left(\mathbb{E} x^{u}, \mathbb{E} p\right)$ based on the assumption

(A1). There is a constant $C \geq 0$ such that

$$
C I_{n \times n}-\left[A_{t}+\bar{A}_{t}-\left(D_{t}+\bar{D}_{t}\right)^{\top} B_{t}^{-1}\left(D_{t}+\bar{D}_{t}\right)\right] \leq 0 .
$$

Hereinafter, $I_{n \times n}$ stands for an $n \times n$ unit matrix.

Noticing the terminal condition of (33), we set

$$
\mathbb{E} p_{t}=\Phi_{t} \mathbb{E} x_{t}^{u}+\Psi_{t}
$$

for two deterministic and differentiable functions $\Phi$ and $\Psi$ such that $\Phi_{T}=H+\bar{H}$ and $\Psi_{T}=L+\bar{L}$. Applying the chain rule for computing the derivative of (34), we have

$$
\begin{aligned}
& \dot{\mathbb{E}} p_{t}=\dot{\Phi}_{t} \mathbb{E} x_{t}^{u}+\Phi_{t} \mathbb{E} \dot{x}_{t}^{u}+\dot{\Psi}_{t} \\
& =\left\{\dot{\Phi}_{t}+\Phi_{t}\left[a_{t}+\bar{a}_{t}-b_{t} B_{t}^{-1}\left(D_{t}+\bar{D}_{t}\right)\right]\right. \\
& \left.-\Phi_{t} b_{t} B_{t}^{-1} b_{t}^{\top} \Phi_{t}\right\} \mathbb{E} x_{t}^{u}+\dot{\Psi}_{t}-\Phi_{t} b_{t} B_{t}^{-1} b_{t}^{\top} \Psi_{t} \\
& +\Phi_{t}\left(\bar{b}_{t}-b_{t} B_{t}^{-1} G_{t}\right) \text {. }
\end{aligned}
$$

Comparing it with the second equation in (33), we deduce a Ricatti equation

$$
\left\{\begin{array}{l}
\dot{\Phi}_{t}+\Phi_{t}\left[a_{t}+\bar{a}_{t}-b_{t} B_{t}^{-1}\left(D_{t}+\bar{D}_{t}\right)\right] \\
+\left[\left(a_{t}+\bar{a}_{t}\right)^{\top}-\left(D_{t}+\bar{D}_{t}\right)^{\top} B_{t}^{-1} b_{t}^{\top}\right] \Phi_{t} \\
-\Phi_{t} b_{t} B_{t}^{-1} b_{t}^{\top} \Phi_{t}+A_{t}+\bar{A}_{t} \\
-\left(D_{t}+\bar{D}_{t}\right)^{\top} B_{t}^{-1}\left(D_{t}+\bar{D}_{t}\right)=0 \\
\Phi_{T}=H+\bar{H}
\end{array}\right.
$$

and an ODE

$$
\left\{\begin{array}{l}
\dot{\Psi}_{t}+\left[\left(a_{t}+\bar{a}_{t}\right)^{\top}-\left(D_{t}+\bar{D}_{t}\right)^{\top} B_{t}^{-1} b_{t}^{\top}\right. \\
\left.-\Phi_{t} b_{t} B_{t}^{-1} b_{t}^{\top}\right] \Psi_{t}+\Phi_{t}\left(\bar{b}_{t}-b_{t} B_{t}^{-1} G_{t}\right) \\
-\left(D_{t}+\bar{D}_{t}\right)^{\top} B_{t}^{-1} G_{t}+F_{t}+\bar{F}_{t}=0, \\
\Psi_{T}=L+\bar{L} .
\end{array}\right.
$$

Clearly, (35) admits a unique solution, and thus, (36) also has a unique solution. Plugging (34) into the first equation of (33), we derive

$$
\left\{\begin{aligned}
\mathbb{E} \dot{x_{t}^{u}}= & {\left[a_{t}+\bar{a}_{t}-b_{t} B_{t}^{-1}\left(D_{t}+\bar{D}_{t}\right)\right.} \\
& \left.-b_{t} B_{t}^{-1} b_{t}^{\top} \Phi_{t}\right] \mathbb{E} x_{t}^{u}-b_{t} B_{t}^{-1} b_{t}^{\top} \Psi_{t} \\
& -b_{t} B_{t}^{-1} G_{t}+\bar{b}_{t}, \\
\mathbb{E} x_{0}^{u}= & \mu_{0},
\end{aligned}\right.
$$

which can be explicitly computed.

Using Theorem 3.3 to (31) with (32), we get the optimal filtering equation

$$
\left\{\begin{aligned}
d \hat{x}_{t}^{u}= & {\left[\left(a_{t}-b_{t} B_{t}^{-1} D_{t}\right) \hat{x}_{t}^{u}-b_{t} B_{t}^{-1} b_{t}^{\top} \hat{p}_{t}+\theta_{1, t}\right] d t } \\
& +\Sigma_{t} f_{t}^{\top}\left(h_{t}^{-1}\right)^{\top} d \bar{w}_{t}, \\
-d \hat{p}_{t}= & {\left[\left(A_{t}-D_{t}^{\top} B_{t}^{-1} D_{t}\right) \hat{x}_{t}^{u}+\left(a_{t}^{\top}-D_{t}^{\top} B_{t}^{-1} b_{t}^{\top}\right) \hat{p}_{t}\right.} \\
& \left.+\theta_{2, t}\right] d t-\hat{\tilde{Q}}_{t} d \bar{w}_{t}, \\
\hat{x}_{0}^{u}= & \mu_{0}, \quad \hat{p}_{T}=H \hat{x}_{T}^{u}+\bar{H} \mathbb{E} x_{T}^{u}+L+\bar{L}
\end{aligned}\right.
$$

with

$$
\theta_{1, t}=\left(\bar{a}_{t}-b_{t} B_{t}^{-1} \bar{D}_{t}\right) \mathbb{E} x_{t}^{u}-b_{t} B_{t}^{-1} G_{t}+\bar{b}_{t},
$$




$$
\begin{aligned}
\theta_{2, t}= & \left(\bar{A}_{t}-D_{t}^{\top} B_{t}^{-1} \bar{D}_{t}-\bar{D}_{t}^{\top} B_{t}^{-1} D_{t}\right. \\
& \left.-\bar{D}_{t}^{\top} B_{t}^{-1} \bar{D}_{t}\right) \mathbb{E} x_{t}^{u}+\left(\bar{a}_{t}^{\top}-\bar{D}_{t}^{\top} B_{t}^{-1} b_{t}^{\top}\right) \mathbb{E} p_{t} \\
& -\left(D_{t}+\bar{D}_{t}\right)^{\top} B_{t}^{-1} G_{t}+F_{t}+\bar{F}_{t},
\end{aligned}
$$

where $\Sigma$ and $\bar{w}$ satisfy (24) and (25), and $\mathbb{E} x^{u}$ and $\mathbb{E} p$ solve (37) and (34), respectively. We assume that the following condition holds.

(A2). There is a constant $C \geq 0$ such that

$$
D_{t}^{\top} B_{t}^{-1} D_{t}-A_{t}-C I_{n \times n} \leq 0 .
$$

Then $(38)$ has a unique solution $\left(\hat{x}^{u}, \hat{p}, \widehat{\widetilde{Q}}\right)$ in the space $\mathscr{L}_{\mathscr{F} Y^{u}}^{2}\left(0, T ; \mathbb{R}^{n+n+n \times \tilde{r}}\right)$ by using Theorem 2.6 in Peng and $\mathrm{Wu}[36]$ again. Similarly, let

$$
\hat{p}_{t}=\Gamma_{t} \hat{x}_{t}^{u}+\Lambda_{t}
$$

for two deterministic and differentiable functions $\Gamma$ and $\Lambda$ such that $\Gamma_{T}=H$ and $\Lambda_{T}=\bar{H} \mathbb{E} x_{T}^{u}+L+\bar{L}$. It follows from Itô's formula that

$$
\begin{aligned}
d \hat{p}_{t}= & \dot{\Gamma}_{t} \hat{x}_{t}^{u} d t+\Gamma_{t} d \hat{x}_{t}^{u}+\dot{\Lambda}_{t} d t \\
= & \left\{\left[\dot{\Gamma}_{t}+\Gamma_{t}\left(a_{t}-b_{t} B_{t}^{-1} D_{t}\right)-\Gamma_{t} b_{t} B_{t}^{-1} b_{t}^{\top} \Gamma_{t}\right] \hat{x}_{t}^{u}\right. \\
& \left.+\Gamma_{t}\left(\theta_{1, t}-b_{t} B_{t}^{-1} b_{t}^{\top} \Lambda_{t}\right)+\dot{\Lambda}_{t}\right\} d t \\
& +\Gamma_{t} \Sigma_{t} f_{t}^{\top}\left(h_{t}^{-1}\right)^{\top} d \bar{w}_{t} .
\end{aligned}
$$

Comparing it with the BSDE in (38), we derive

$$
\left\{\begin{array}{l}
\dot{\Gamma}_{t}+\Gamma_{t}\left(a_{t}-b_{t} B_{t}^{-1} D_{t}\right)+\left(a_{t}^{\top}-D_{t}^{\top} B_{t}^{-1} b_{t}^{\top}\right) \Gamma_{t} \\
-\Gamma_{t} b_{t} B_{t}^{-1} b_{t}^{\top} \Gamma_{t}+A_{t}-D_{t}^{\top} B_{t} D_{t}=0, \\
\Gamma_{T}=H
\end{array}\right.
$$

and

$$
\left\{\begin{array}{l}
\dot{\Lambda}_{t}+\left(a_{t}^{\top}-D_{t}^{\top} B_{t}^{-1} b_{t}^{\top}-\Gamma_{t} b_{t} B_{t}^{-1} b_{t}^{\top}\right) \Lambda_{t} \\
+\Gamma_{t} \theta_{1, t}+\theta_{2, t}=0 \\
\Lambda_{T}=\bar{H} \mathbb{E} x_{T}^{u}+L+\bar{L}
\end{array}\right.
$$

which have a unique solution, respectively. Substituting (39) into (32), we get

$$
u_{t}=-B_{t}^{-1}\left[\left(b_{t}^{\top} \Gamma_{t}+D_{t}\right) \hat{x}_{t}^{u}+\bar{D}_{t} \mathbb{E} x_{t}^{u}+b_{t}^{\top} \Lambda_{t}+G_{t}\right],
$$

where $\mathbb{E} x^{u}, \Gamma, \Lambda$ and $\hat{x}^{u}$ solve (37), (40), (41) and the closed-loop system

$$
\left\{\begin{aligned}
d \hat{x}_{t}^{u}= & \left\{\left[a_{t}-b_{t} B_{t}^{-1}\left(D_{t}+b_{t}^{\top} \Gamma_{t}\right)\right] \hat{x}_{t}^{u}\right. \\
& \left.-b_{t} B_{t}^{-1} b_{t}^{\top} \Lambda_{t}+\theta_{1, t}\right\} d t+\Sigma_{t} f_{t}^{\top}\left(h_{t}^{-1}\right)^{\top} d \bar{w}_{t}, \\
\hat{x}_{0}^{u}= & \mu_{0}
\end{aligned}\right.
$$

respectively.

Step 4: (42) is the optimal control.

According to $(25), \bar{w}_{t}$ is an $\mathscr{F}_{t}^{Y^{0}}$-adapted standard Brownian motion. Then it is easy to see from (43) that $\hat{x}_{t}^{u}$ is $\mathscr{F}_{t}^{Y^{0}}$-adapted, and hence, $u_{t}$ given by $(42)$ is $\mathscr{F}_{t} Y^{0}$ adapted. On the other hand, applying Itô's formula to $\left\langle\hat{x}^{u}, \hat{x}^{u}\right\rangle$ with Burkholder-Davis-Gundy inequality, we deduce

$$
\mathbb{E} \sup _{0 \leq t \leq T}\left|\hat{x}_{t}^{u}\right|^{2}<+\infty .
$$

Then $u \in \mathscr{U}_{a d}^{0}$. Next, we will prove that $u_{t}$ is also $\mathscr{F}_{t} Y^{u}$. adapted. If so, then $u \in \mathscr{U}_{a d}$, and consequently, $u$ is optimal via Theorem 3.2. In fact, using (25) again, (43) can be rewritten as

$$
\left\{\begin{aligned}
d \hat{x}_{t}^{u}= & \left\{\left[a_{t}-b_{t} B_{t}^{-1}\left(D_{t}+b_{t}^{\top} \Gamma_{t}\right)\right] \hat{x}_{t}^{u}\right. \\
& \left.-b_{t} B_{t}^{-1} b_{t}^{\top} \Lambda_{t}+\theta_{1, t}\right\} d t \\
& +\Sigma_{t} f_{t}^{\top}\left(h_{t}^{-1}\right)^{\top} h_{t}^{-1}\left[d Y_{t}^{u}\right. \\
& \left.-\left(f_{t} \hat{x}_{t}^{u}+\bar{f}_{t} \mathbb{E} x_{t}^{u}+g_{t}\right) d t\right], \\
\hat{x}_{0}^{u}= & \mu_{0} .
\end{aligned}\right.
$$

From the optimal filtering equation, it is easy to check that $\hat{x}_{t}^{u}$ is $\mathscr{F}_{t}^{Y^{u}}$-adapted, so is $u_{t}$. Then $u \in \mathscr{U}_{a d}$. Therefore, the claim holds.

Step 5: Optimal cost functional.

Since the solution $\Sigma$ of (24) is independent of $v$, the optimal cost functional is rewritten as

$$
\begin{aligned}
J[u]= & J_{1}[u]+\int_{0}^{T} N^{\top} \chi_{0}^{t} \bar{\psi}_{t} d t+\frac{1}{2} \int_{0}^{T} \operatorname{tr}\left(A_{t} \Sigma_{t}\right) d t \\
& +\operatorname{tr}\left(H \Sigma_{T}\right)
\end{aligned}
$$

with

$$
\begin{aligned}
J_{1}[u]= & \frac{1}{2} \mathbb{E}\left\{\int _ { 0 } ^ { T } \left[\left\langle A_{t} \hat{x}_{t}^{u}, \hat{x}_{t}^{u}\right\rangle+\left\langle\bar{A}_{t} \mathbb{E} x_{t}^{u}, \mathbb{E} x_{t}^{u}\right\rangle\right.\right. \\
& +\left\langle B_{t} u_{t}, u_{t}\right\rangle+2\left\langle D_{t} \hat{x}_{t}^{u}, u_{t}\right\rangle+2\left\langle\bar{D}_{t} \mathbb{E} x_{t}^{u}, u_{t}\right\rangle \\
& \left.+2\left\langle F_{t}, x_{t}^{u}\right\rangle+2\left\langle\bar{F}_{t}, \mathbb{E} x_{t}^{u}\right\rangle+2\left\langle G_{t}, u_{t}\right\rangle\right] d t \\
& +\left\langle H \hat{x}_{T}^{u}, \hat{x}_{T}^{u}\right\rangle+\left\langle\bar{H} \mathbb{E} x_{T}^{u}, \mathbb{E} x_{T}^{u}\right\rangle+2\left\langle L, x_{T}^{u}\right\rangle \\
& \left.+2\left\langle\bar{L}, \mathbb{E} x_{T}^{u}\right\rangle\right\} .
\end{aligned}
$$

Here $\hat{x}^{u}$ solves (43), and $\operatorname{tr}(A)$ denotes the trace of the 
matrix $A$. Using Itô's formula, we deduce

$$
\begin{aligned}
d\left\langle\Gamma_{t} \hat{x}_{t}^{u}, \hat{x}_{t}^{u}\right\rangle= & \left\langle\left(D_{t}^{\top} B_{t} D_{t}-A_{t}-\Gamma_{t} b_{t} B_{t}^{-1} b_{t}^{\top} \Gamma_{t}\right) \hat{x}_{t}^{u}\right. \\
& \left.-2 \Gamma_{t} b_{t} B_{t}^{-1} b_{t}^{\top} \Lambda_{t}+2 \Gamma_{t} \theta_{1, t}, \hat{x}_{t}^{u}\right\rangle d t \\
& +\left\langle\Sigma_{t} f_{t}^{\top}\left(h_{t}^{-1}\right)^{\top}, \Gamma_{t} \Sigma_{t} f_{t}^{\top}\left(h_{t}^{-1}\right)^{\top}\right\rangle d t \\
& +\left\langle\Gamma_{t} \Sigma_{t} f_{t}^{\top}\left(h_{t}^{-1}\right)^{\top} d \bar{w}_{t}, \hat{x}_{t}^{u}\right\rangle \\
& +\left\langle\Gamma_{t} \hat{x}_{t}^{u}, \Sigma_{t} f_{t}^{\top}\left(h_{t}^{-1}\right)^{\top} d \bar{w}_{t}\right\rangle .
\end{aligned}
$$

Then

$$
\begin{aligned}
& \mathbb{E}\left\langle H \hat{x}_{T}^{u}, \hat{x}_{T}^{u}\right\rangle \\
= & \mathbb{E} \int_{0}^{T}\left\langle\left(D_{t}^{\top} B_{t} D_{t}-A_{t}-\Gamma_{t} b_{t} B_{t}^{-1} b_{t}^{\top} \Gamma_{t}\right) \hat{x}_{t}^{u}\right. \\
& \left.-2 \Gamma_{t} b_{t} B_{t}^{-1} b_{t}^{\top} \Lambda_{t}+2 \Gamma_{t} \theta_{1, t}, \hat{x}_{t}^{u}\right\rangle d t \\
& +\int_{0}^{T}\left\langle\Sigma_{t} f_{t}^{\top}\left(h_{t}^{-1}\right)^{\top}, \Gamma_{t} \Sigma_{t} f_{t}^{\top}\left(h_{t}^{-1}\right)^{\top}\right\rangle d t \\
& +\left\langle\Gamma_{0} \mu_{0}, \mu_{0}\right\rangle .
\end{aligned}
$$

Similarly, applying Itô's formula to $\left\langle\Lambda, \hat{x}^{u}\right\rangle$, we get

$$
\begin{aligned}
& \mathbb{E}\left\langle\bar{H} \mathbb{E} x_{T}^{u}+L+\bar{L}, \hat{x}_{T}^{u}\right\rangle \\
= & \int_{0}^{T}\left\langle\Lambda_{t}, \theta_{1, t}-b_{t} B_{t}^{-1} b_{t}^{\top} \Lambda_{t}\right\rangle d t+\left\langle\Lambda_{0}, e_{0}\right\rangle \\
& -\mathbb{E} \int_{0}^{T}\left\langle\Gamma_{t} \theta_{1, t}+\theta_{2, t}, \hat{x}_{t}^{u}\right\rangle d t .
\end{aligned}
$$

Plugging (42), (45) and (46) into (44), we derive

$$
\begin{aligned}
J[u]= & \frac{1}{2}\left\{\int _ { 0 } ^ { T } \left\langle\left[\left(2 D_{t}+\bar{D}_{t}\right)^{\top} B_{t}^{-1} \bar{D}_{t}-\bar{A}_{t}\right.\right.\right. \\
& \left.\left.\left.-2 \bar{a}_{t}^{\top} \Gamma_{t}\right] \mathbb{E} x_{t}^{u}, \mathbb{E} x_{t}^{u}\right\rangle d t-\left\langle\bar{H} \mathbb{E} x_{T}^{u}, \mathbb{E} x_{T}^{u}\right\rangle\right\} \\
& +\int_{0}^{T}\left\langle\bar{D}_{t}^{\top} B_{t}^{-1} b_{t}^{\top} \Gamma_{t}-\Gamma_{t} b_{t}^{\top} B_{t}^{-1} G_{t}\right. \\
& \left.-\Gamma_{t} b_{t} B_{t}^{-1} b_{t}^{\top} \Lambda_{t}, \mathbb{E} x_{t}^{u}\right\rangle d t \\
& +\int_{0}^{T}\left\langle b_{t}^{\top} \Gamma_{t}, B_{t}^{-1}\left(b_{t}^{\top} \Lambda_{t}+G_{t}\right)\right\rangle d t \\
& +\frac{1}{2} \int_{0}^{T}\left\langle\Lambda_{t}, 2 \bar{b}_{t}-2 b_{t} B_{t}^{-1} G_{t}-b_{t} B_{t}^{-1} b_{t}^{\top} \Lambda_{t}\right\rangle d t \\
& -\frac{1}{2} \int_{0}^{T}\left\langle G_{t}, B_{t}^{-1} G_{t}\right\rangle d t \\
& +\frac{1}{2}\left\langle\Gamma_{0} \mu_{0}, \mu_{0}\right\rangle+\left\langle\Lambda_{0}, \mu_{0}\right\rangle \\
& +\frac{1}{2} \int_{0}^{T}\left\langle\Sigma_{t} f_{t}^{\top}\left(h_{t}^{-1}\right)^{\top}, \Gamma_{t} \Sigma_{t} f_{t}^{\top}\left(h_{t}^{-1}\right)^{\top}\right\rangle d t \\
& +\int_{0}^{T} N^{\top} \chi_{0}^{t} \bar{\psi}_{t} d t+\frac{1}{2} \int_{0}^{T} t r\left(A_{t} \Sigma_{t}\right) d t \\
& +t r\left(H \Sigma_{T}\right) .
\end{aligned}
$$

We summarize the above deduction as follows.

Proposition 4.2. Under the assumptions (A1), (A2), $M=0$, and $\bar{\beta}_{t}=\gamma_{t}=\bar{\gamma}_{t}=\tilde{\gamma}_{t}=\overline{\tilde{\gamma}}_{t}=0$, the optimal feedback control and the optimal cost functional of Problem (MFLQ) are explicitly given by (42) and (47), respectively.

\section{Solution to Problem (AL)}

In this section, we are interested in explicitly computing Problem (AL) introduced in Section 1.2 with the assumption that the generator

$$
G(t, y, z)=\beta_{t} y+\psi_{t} v
$$

where $\beta$ and $\psi$ are deterministic and uniformly bounded. With the generator, Problem (AL) is a special case of Problem (MFLQ). (33) is reduced to a decoupled forward-backward ODE

$$
\left\{\begin{aligned}
\mathbb{E} \dot{x_{t}^{u}}= & \left(a_{t}+\bar{a}_{t}\right) \mathbb{E} x_{t}^{u}-B_{t}^{-1} b_{t}^{2} \mathbb{E} p_{t} \\
& +N B_{t}^{-1} b_{t} \psi_{t} e^{\int_{0}^{t} \beta_{s} d s}+\bar{b}_{t}, \\
\dot{\mathbb{E} p_{t}=} & -\left(a_{t}+\bar{a}_{t}\right) \mathbb{E} p_{t}, \\
\mathbb{E} x_{0}^{u}= & \mu_{0}, \quad \mathbb{E} p_{T}=-N e^{\int_{0}^{T} \beta_{t} d t} .
\end{aligned}\right.
$$

Solving it, we get

$$
\mathbb{E} p_{t}=-N e^{\int_{0}^{T} \beta_{t} d t+\int_{t}^{T}\left(a_{s}+\bar{a}_{s}\right) d s}
$$

and

$$
\begin{aligned}
\mathbb{E} x_{t}^{u}= & \mu_{0} e^{\int_{0}^{t}\left(a_{s}+\bar{a}_{s}\right) d s}-\int_{0}^{t} B_{s}^{-1} b_{s}^{2} e^{\int_{s}^{t}\left(a_{r}+\bar{a}_{r}\right) d r} \mathbb{E} p_{s} d s \\
& +\int_{0}^{t}\left(\bar{b}_{s}+N B_{s}^{-1} b_{s} \psi_{s} e^{\int_{0}^{s} \beta_{r} d r}\right) e^{\int_{s}^{t}\left(a_{r}+\bar{a}_{r}\right) d r} d s,
\end{aligned}
$$

where $\mathbb{E} p$ is determined by (48). (40) and (41) are rewritten as

$$
\left\{\begin{array}{l}
\dot{\Gamma}_{t}+2 a_{t} \Gamma_{t}-B_{t}^{-1} b_{t}^{2} \Gamma_{t}^{2}=0 \\
\Gamma_{T}=H
\end{array}\right.
$$

and

$$
\left\{\begin{array}{l}
\dot{\Lambda}_{t}+\left(a_{t}-B_{t}^{-1} b_{t}^{2} \Gamma_{t}\right) \Lambda_{t}+\Gamma_{t} \theta_{1, t}+\theta_{2, t}=0, \\
\Lambda_{T}=-H \mathbb{E} x_{T}^{u}-N e^{\int_{0}^{T} \beta_{t} d t},
\end{array}\right.
$$

where

$\theta_{1, t}=\bar{a}_{t} \mathbb{E} x_{t}^{u}+B_{t}^{-1} b_{t} \psi_{t} N e^{\int_{0}^{t} \beta_{s} d s}+\bar{b}_{t}, \quad \theta_{2, t}=\bar{a}_{t} \mathbb{E} p_{t}$, 
with $\mathbb{E} p$ and $\mathbb{E} x$ be determined by (48) and (49). This gives

$$
\begin{aligned}
\Lambda_{t}= & -\left(H \mathbb{E} x_{T}^{u}+N e^{\int_{0}^{T} \beta_{s} d s}\right) e^{\int_{t}^{T}\left(a_{s}-B_{s}^{-1} b_{s}^{2} \Gamma_{s}\right) d s} \\
& +\int_{t}^{T}\left(\Gamma_{s} \theta_{1, s}+\theta_{2, s}\right) e^{\int_{t}^{s}\left(a_{r}-B_{r}^{-1} b_{r}^{2} \Gamma_{r}\right) d r} d s .
\end{aligned}
$$

Then the optimal control strategy in a feedback form is

$$
u_{t}=-B_{t}^{-1}\left[b_{t}\left(\Gamma_{t} \hat{x}_{t}^{u}+\Lambda_{t}\right)-N \psi_{t} e_{0}^{\int_{0}^{t} \beta_{s} d s}\right] .
$$

Here $\Gamma$ and $\Lambda$ solve (50) and (51). The filtered cashbalance $\hat{x}^{u}$ satisfies a closed-loop system

$$
\left\{\begin{aligned}
d \hat{x}_{t}^{u}= & {\left[\left(a_{t}-B_{t}^{-1} b_{t}^{2} \Gamma_{t}\right) \hat{x}_{t}^{u}-B_{t}^{-1} b_{t}^{2} \Lambda_{t}+\theta_{1, t}\right] d t } \\
& +\Sigma_{t} f_{t} h_{t}^{-1} d \bar{w}_{t}, \\
\hat{x}_{0}^{u}= & \mu_{0}
\end{aligned}\right.
$$

with $\Sigma$ being governed by

$$
\left\{\begin{array}{l}
\dot{\Sigma}_{t}-2 a_{t} \Sigma_{t}+h_{t}^{-2} f_{t}^{2} \Sigma_{t}^{2}-c_{t}^{2}=0 \\
\Sigma_{0}=\sigma_{0}
\end{array}\right.
$$

The remaining part of this section is devoted to illustrating the above results via numerical computations. For the sake of illustrations, let the time granularity for all the market parameters be yearly. Suppose that the initial investment $\xi=1$, the discount rate $a_{t}=0.03$, the liability rate $\bar{b}_{t}=0.01$, and the volatility rates $c_{t}=0.04$ and $h_{t}=0.1$. We further suppose that the market parameters $\bar{a}_{t}=0.03, \beta_{t}=0.06, f_{t}=0.1, b_{t}=\psi_{t}=B_{t}=$ $N=1$, and $H=0.01$. With the data, it is easy to see that

$$
\begin{aligned}
\mathbb{E} p_{t}= & -e^{0.06(2-t)}, \\
\mathbb{E} x_{t}^{u}= & e^{0.06 t}\left[1+t+\frac{25}{3} e^{0.12}\left(1-e^{-0.12 t}\right)\right. \\
& \left.+\frac{1}{6}\left(1-e^{-0.06 t}\right)\right], \\
\theta_{1, t}= & 0.03 \mathbb{E} x_{t}^{u}+e^{0.06 t}+0.01, \quad \theta_{2, t}=-0.03 e^{0.06(2-t)}, \\
\Gamma_{t}= & \frac{0.06 e^{0.06(1-t)}}{5+e^{0.06(1-t)}},
\end{aligned}
$$

and

$$
\begin{aligned}
\Lambda_{t}= & -\left(0.01 \mathbb{E} x_{1}^{u}+e^{0.06}\right) e^{\int_{t}^{1}\left(0.03-\Gamma_{s}\right) d s} \\
& +\int_{t}^{1}\left(\Gamma_{s} \theta_{1, s}+\theta_{2, s}\right) e^{\int_{t}^{s}\left(0.03-\Gamma_{r}\right) d r} d s .
\end{aligned}
$$

Then the optimal control strategy of the firm is

$$
u_{t}=-\left(\Gamma_{t} \hat{x}_{t}^{u}+\Lambda_{t}-e^{0.03 t}\right),
$$

where the optimal filtering of the cash-balance $x^{u}$ satisfies

$$
\left\{\begin{aligned}
d \hat{x}_{t}^{u} & =\left[\left(0.03-\Gamma_{t}\right) \hat{x}_{t}^{u}-\Lambda_{t}+\theta_{1, t}\right] d t+\Sigma_{t} d \bar{w}_{t}, \\
\hat{x}_{0}^{u} & =1
\end{aligned}\right.
$$

with

$$
\Sigma_{t}=\frac{0.08\left(e^{0.1 t}-1\right)}{e^{0.1 t}-4}
$$

$\Gamma, \Lambda$ and $\theta_{1}$ being determined above.

\section{Concluding remarks}

This article studies an optimal control problem for meanfield FBSDE with noisy observation. Since mean-field FBSDE and optimal filtering are considered, the control problem has been basically unexplored so far. The control problem covers more models in reality, but causes a trouble in solving the problem. The backward separation method with the decomposition of the state and the observation is further developed, and is introduced to overcome the resulting difficulty. These results obtained in this article improve the first author's previous works $[39,40,41,42]$, and are helpful for studying meanfield game for FBSDE and systematic risk model with noisy observation. The details of how we study these problems will be presented elsewhere.

Let us now make several remarks in order to close this section. (1) In most optimal control problems for meanfield stochastic systems, we assume that all coefficients of the optimal control problems are deterministic. Otherwise, there is an immediate difficulty to study the problems. One reason is that the key equality $\mathbb{E}\left(a_{t} x_{t}\right)=$ $a_{t} \mathbb{E} x_{t}$ is no longer true if $a_{t}$ is also a stochastic process. But some special cases with stochastic coefficients, say, Example 2.1, can be solved by a simple reduction method. Then it is natural to ask if the method is applicable for slightly more complicated cases. We hope to answer it in the near future. (2) Similar to Example 2.2, Problem (MFLQ) can also be reduced to an LQ problem for non-standard FBSDE with control set constraint. This motivates us to investigate such a class of LQ problems for non-standard FBSDEs in the future. In return, it will be helpful to study LQ problems for meanfield FBSDEs. (3) The solution of the BSDE in (7) is a non-Gaussian process in general, and thus, the optimal filter of the BSDE is infinite. Then it is highly desirable to study the numerical approximation of the optimal filter and the optimal control in our future publications. 


\section{Acknowledgements}

The authors would like to thank the editors and three anonymous referees for their constructive and insightful comments for improving the quality of this work.

\section{Appendix}

We present three lemmas first, and then give a proof of Theorem 2.1.

Lemma A.1. For any $v_{j} \in \mathscr{L}_{\mathscr{F}}^{2}\left(0, T ; \mathbb{R}^{k}\right), j=1,2$, there is a constant $C>0$ such that

$$
\begin{aligned}
& \mathbb{E} \sup _{0 \leq t \leq T}\left|x_{t}^{v_{1}}-x_{t}^{v_{2}}\right|^{2} \leq C \mathbb{E} \int_{0}^{T}\left|v_{1, t}-v_{2, t}\right|^{2} d t, \\
& \mathbb{E} \sup _{0 \leq t \leq T}\left|y_{t}^{v_{1}}-y_{t}^{v_{2}}\right|^{2} \\
& +\mathbb{E} \int_{0}^{T}\left(\left|z_{t}^{v_{1}}-z_{t}^{v_{2}}\right|^{2}+\left|\tilde{z}_{t}^{v_{1}}-\tilde{z}_{t}^{v_{2}}\right|^{2}\right) d t \\
& \leq C\left(\mathbb{E}\left|x_{T}^{v_{1}}-x_{T}^{v_{2}}\right|^{2}+\int_{0}^{T} \sup _{0 \leq s \leq t} \mathbb{E}\left|x_{s}^{v_{1}}-x_{s}^{v_{2}}\right|^{2} d t\right. \\
& \left.\quad+\mathbb{E} \int_{0}^{T}\left|v_{1, t}-v_{2, t}\right|^{2} d t\right) .
\end{aligned}
$$

Proof. These two estimates can be derived by Itô's formula, Gronwall's inequality and Burkholder-DavisGundy inequality. We omit the proof for simplicity.

Lemma A.2. For any $v, v_{j} \in \mathscr{U}_{a d}(j=1,2, \cdots)$ satisfying $v_{j} \rightarrow v$ in $\mathscr{L}_{\mathscr{F}}^{2}\left(0, T ; \mathbb{R}^{k}\right)$, it holds

$$
\lim _{j \rightarrow+\infty} J\left[v_{j}\right]=J[v] .
$$

Proof. Using Taylor's expansion, Hölder's inequality and Lemma A.1, we deduce

$$
\begin{aligned}
& \quad\left|\mathbb{E} \int_{0}^{T} l\left(\Theta_{t}^{v_{j}}\right) d t-\mathbb{E} \int_{0}^{T} l\left(\Theta_{t}^{v}\right) d t\right| \\
& \leq C \\
& \quad+\mathbb{E} \int_{0}^{T}\left(1+\left|x_{t}^{v_{j}}\right|+\left|x_{t}^{v}\right|+\mathbb{E}\left|x_{t}^{v_{j}}\right|+\mathbb{E}\left|x_{t}^{v}\right|\right. \\
& \left.\quad+\left|v_{j, t}\right|+\left|v_{t}\right|\right)\left(\left|x_{t}^{v_{j}}-x_{t}^{v}\right|+\left|\mathbb{E} x_{t}^{v_{j}}-\mathbb{E} x_{t}^{v}\right|\right. \\
& \leq C \\
& \quad \sqrt{\mathbb{E} \int_{0}^{T} \aleph_{t}^{v, v_{j}} d t\left(\sqrt{\mathbb{E} \sup _{0 \leq t \leq T}\left|x_{t}^{v_{j}}-x_{t}^{v}\right|^{2}}\right.} \\
& \left.\quad+\sqrt{\mathbb{E} \int_{0}^{T}\left|v_{j, t}-v_{t}\right|^{2} d t}\right) \rightarrow 0
\end{aligned}
$$

as $j \rightarrow+\infty$, where $C>0$ is a constant, and

$$
\begin{aligned}
\aleph_{t}^{v, v_{j}}= & 1+\left|x_{t}^{v_{j}}\right|^{2}+\left|x_{t}^{v}\right|^{2}+\mathbb{E}\left|x_{t}^{v_{j}}\right|^{2} \\
& +\mathbb{E}\left|x_{t}^{v}\right|^{2}+\left|v_{j, t}\right|^{2}+\left|v_{t}\right|^{2} .
\end{aligned}
$$

In a same way, we have

$$
\mathbb{E} \phi\left(\Xi_{T}^{v_{j}}\right) \rightarrow \mathbb{E} \phi\left(\Xi_{T}^{v}\right), \quad \mathbb{E} \varphi\left(y_{0}^{v_{j}}\right) \rightarrow \mathbb{E} \varphi\left(y_{0}^{v}\right)
$$

with $j \rightarrow+\infty$. Then the proof is complete.

Lemma A.3. $\mathscr{U}_{a d}$ is dense in $\mathscr{U}_{a d}^{0}$.

Proof. For any $v \in \mathscr{U}_{a d}^{0}$, define a family of controls by

$$
v_{j t}= \begin{cases}\nu, & \text { for } 0 \leq t \leq \delta_{j}, \\ \frac{1}{\delta_{j}} \int_{(i-1) \delta_{j}}^{i \delta_{j}} v_{s} d s, & \text { for } i \delta_{j}<t \leq(i+1) \delta_{j},\end{cases}
$$

where $\nu \in U, i, j$ are natural numbers, $1 \leq i \leq j-1$, and $\delta_{j}=T / j$. Similar to Bensoussan [2], we can prove that (i) $v_{j} \in \mathscr{U}_{a d}$ for any $j$, and (ii) $v_{j} \rightarrow v$ as $j \rightarrow+\infty$ in $\mathscr{L}_{\mathscr{F} Y^{0}}^{2}(0, T ; U)$. Then it implies the desired result.

Proof of Theorem 2.1. From Definition 2.1, we have $\mathscr{U}_{a d} \subseteq \mathscr{U}_{a d}^{0}$, and thus, $\inf _{v^{\prime} \in \mathscr{U}_{a d}} J\left[v^{\prime}\right] \geq \inf _{v \in \mathscr{U}_{a d}^{0}} J[v]$. On the other hand, since $v_{j}$ defined in the proof of Lemma A.3 is an element of $\mathscr{U}_{a d}$, then $\inf _{v^{\prime} \in \mathscr{U}_{a d}} J\left[v^{\prime}\right] \leq$ $J\left[v_{j}\right]$, and consequently, it follows from Lemma A.2 that $\inf _{v^{\prime} \in \mathscr{U}_{a d}} J\left[v^{\prime}\right] \leq \lim _{j \rightarrow+\infty} J\left[v_{j}\right]=J[v]$. Due to the arbitrariness of $v$, then $\inf _{v^{\prime} \in \mathscr{U}_{a d}} J\left[v^{\prime}\right] \leq \inf _{v \in \mathscr{U}_{a d}^{0}} J[v]$. Thus, the proof is complete.

\section{References}

[1] Ahmed, N.U., \& Ding, X. (2001). Controlled McKean-Vlasov equation. Commun. Appl. Anal., 5, 183-206.

[2] Bensoussan, A. (1982). Stochastic control by functional analysis methods. New York: North-Holland Publishing Company.

[3] Bensoussan, A. (1992). Stochastic control of partially observable systems. Cambridge: Cambridge University Press.

[4] Bensoussan, A., Frehse, J., \& Yam, P. (2013). Mean field games and mean field type control theory. New York: SpringerVerlag.

[5] Bensoussan, A., Yam, S. C. P., \& Zhang, Z. (2015). Wellposedness of mean-field type forward-backward stochastic differential equations. Stoch. Proc. Appl., 125, 3327-3354.

[6] Bensoussan, A., Sung, K. C. J., Yam, S. C. P., \& Yung, S. P. (2016). Linear-quadratic mean-field games. J. Optim. Theory Appl., 169, 496-529.

[7] Buckdahn, R., Djehiche, B., \& Li, J. (2011). A general stochastic maximum principle for SDEs of mean-field type. Appl. Math. Optim., 64, 197-216.

[8] Buckdahn, R., Djehiche, B., Li, J., \& Peng, S. (2009). Mean-field backward stochastic differential equations: a limit approach. Ann. Probab., 37, 1524-1565. 
[9] Carmona, R., \& Delarue, F. (2015). Forward-backward stochastic differential equations and controlled McKeanVlasov dynamics. Ann. Probab., 43, 2647-2700.

[10] Carmona, R., Delarue, F., \& Lachapelle, A. (2013). Control of McKean-Vlasov dynamics versus mean field games. Math. Financ. Econ., 7, 131-166.

[11] Carmona, R., Fouque, J., \& Sun, L. (2015). Mean field games and systemic risk. Commun. Math. Sci., 13, 911-933.

[12] Djehiche, B., Tembine, H., \& Tempone, R. (2015). Stochastic maximum principle for risk-sensitive mean-field type control. IEEE Trans. Automat. Control, 60, 2640-2649.

[13] Duffie, D., \& Epstein, L. (1992). Stochastic differential utility. Econometrica, 60, 353-394.

[14] El Karoui, N., Peng, S., \& Quenez, M. C. (1997). Backward stochastic differential equations in finance. Math. Financ., 7, $1-71$.

[15] Elliott, R., Li, X., \& Ni, Y. (2013). Discrete time meanfield stochastic linear quadratic optimal control problems. Automatica, 49, 3222-3233.

[16] Hafayed, M., \& Abbas, S. (2014). On near-optimal meanfield stochastic singular controls: necessary and sufficient conditions for near-optimality. J. Optim. Theory Appl., 160, 778-808.

[17] Hafayed, M., Abba, A., \& Abbas, S. (2016). On partialinformation optimal singular control problem for meanfield stochastic differential equations driven by Teugels martingales measures. Intern. J. Control, 89, 397-410.

[18] Hafayed, M., Abba, S., \& Abbas, A. (2015). On mean-field partial information maximum principle of optimal control for stochastic systems with L'evy processes. J. Optim. Theory Appl., 167, 1051-1069.

[19] Hafayed, M., Tabet, \& Boukaf, S. (2015). Mean-field maximum principle for optimal control of forward-backward stochastic systems with jumps and its application to meanvariance portfolio problem. Commun. Math. Stat., 3, 163-186.

[20] Hu, Y., Nualart, D., \& Zhou, Q. (2014). On optimal meanfield type control problems of stochastic systems with jump processes under partial information. arXiv:1403.4377v1.

[21] Huang, J., Li, X., \& Yong, J. (2015). A linear-quadratic optimal control problem for mean-field stochastic differential equations in infinite horizon. Math. Control Relat. Fields, 5, 97-139.

[22] Huang, J., Wang, G., \& Wu, Z. (2010). Optimal premium policy of an insurance firm: full and partial information. Insur. Math. Econ., 47, 208-215.

[23] Huang, J., Wang, S., \& Wu, Z. (2016). Backward meanfield linear-quadratic-Gaussian (LQG) games: full and partial information. IEEE Trans. Automat. Control, DOI: 10.1109/TAC.2016.2519501.

[24] Huang, M., Caines, P., \& Malhamé, R. (2006). Distributed multi-agent decision-making with partial observations: asymptotic Nash equilibria. Proceedings of the 17th International Symposium on Mathematial Theory of Networks and Systems, Kyoto, Japan (pp. 2725-2730).

[25] Huang, M., Caines, P., \& Malhamé, R. (2007). Largepopulation cost-coupled LQG problems with nonuniform agents: individual-mass behavior and decentralized $\varepsilon$-Nash equilibria. IEEE Trans. Automat. Control, 52, 1560-1571.

[26] Kac, M. (1956). Foundations of kinetic theory. Proceedings of the 3rd Berkeley Symposium on Mathematical Statistics and Probability, 3, 171-197.

[27] Lasry, J., \& Lions, P. (2007). Mean field games. Japan. J. Math., 2, 229-260.
[28] Li, J. (2012). Stochastic maximum principle in the mean-field controls. Automatica, 48, 366-373.

[29] Li, R., \& Liu, B. (2014). A maximum principle for fully coupled stochastic control systems of mean-field type. J. Math. Anal. Appl., 415, 902-930.

[30] Liptser, R. S., \& Shiryayev, A. N. (1977). Statistics of random processes. New York: Springer-Verlag.

[31] McKean, H. P. (1967). Propagation of chaos for a class of nonlinear parabolic equations. Lecture Series in Differential Equations, 7, 41-57.

[32] Meyer-Brandis, T., Øksendal, B., \& Zhou, X. (2012). A meanfield stochastic maximum principle via Malliavin calculus. Stochastics, 84, 643-666.

[33] Ni, Y., Elliott, R., \& Li, X. (2015). Discrete-time meanfield stochastic linear-quadratic optimal control problems, II: Infinite horizon case. Automatica, 57, 65-77.

[34] Ni, Y., Zhang, J., \& Li, X. (2015). Indefinite mean-field stochastic linear-quadratic optimal control. IEEE Trans. Automat. Control, 60, 1786-1800.

[35] Norberg, R. (1999). Ruin problems with assets and liabilities of diffusion type. Stoch. Proc. Appl., 81, 255-269.

[36] Peng, S., \& Wu, Z. (1999). Fully coupled forward-backward stochastic differential equations and applications to optimal control. SIAM J. Control Optim., 37, 825-843.

[37] Shen, Y., Meng, Q., \& Shi, P. (2014). Maximum principle for mean-field jump-diffusion stochastic delay differential equations and its application to finance. Automatica, 50, 1565-1579.

[38] Tembine, H., Zhu, Q., \& Basar, T. (2014). Risk-sensitive mean-field games. IEEE Trans. Automat. Control, 59, 835850.

[39] Wang, G., Wu, Z., \& Xiong, J. (2013). Maximum principles for forward-backward stochastic control systems with correlated state and observation noise. SIAM J. Control Optim., 51, 491-524.

[40] Wang, G., Wu, Z., \& Xiong, J. (2015). A linear-quadratic optimal control problem of forward-backward stochastic differential equations with partial information. IEEE Trans. Automat. Control, 60, 2904-2916.

[41] Wang, G., Wu, Z., \& Zhang, C. (2014). Maximum principles for partially observed mean-field stochastic systems with applications to financial engineering. Proceedings of the 33rd Chinese Control Conference, Nanjing, China (pp. 5357-5362).

[42] Wang, G., Zhang, C., \& Zhang, W. (2014). Stochastic maximum principle for mean-field type optimal control with partial information. IEEE Trans. Automat. Control, 59, 522528.

[43] Yong, J. (2013). Linear-quadratic optimal control problems for mean-field stochastic differential equations. SIAM J. Control Optim., 51, 2809-2838.

[44] Yong, J. (2013). Linear-quadratic optimal control problems for mean-field stochastic differential equations-time-consistent solutions. arXiv: 1304.3964v2.

[45] Zhang, H. (2016). A necessary condition for mean-field type stochastic differential equations with correlated state and observation noises. J. Ind. Manag. Optim., 12, 1287-1301. 